\title{
Polymer dynamics in bimodal polyethylene melts: A study with neutron spin echo spectroscopy and pulsed field gradient nuclear magnetic resonance
}

S. Rathgeber, L. Willner, D. Richter, A. Brulet, B. Farago, M. Appel, and G. Fleischer

Citation: The Journal of Chemical Physics 110, 10171 (1999); doi: 10.1063/1.478890

View online: https://doi.org/10.1063/1.478890

View Table of Contents: http://aip.scitation.org/toc/jcp/110/20

Published by the American Institute of Physics

\section{Articles you may be interested in}

A Theory of the Linear Viscoelastic Properties of Dilute Solutions of Coiling Polymers

The Journal of Chemical Physics 21, 1272 (1953); 10.1063/1.1699180

Polymer dynamics under cylindrical confinement featuring a locally repulsive surface: A quasielastic neutron scattering study

The Journal of Chemical Physics 146, 203306 (2017); 10.1063/1.4974836

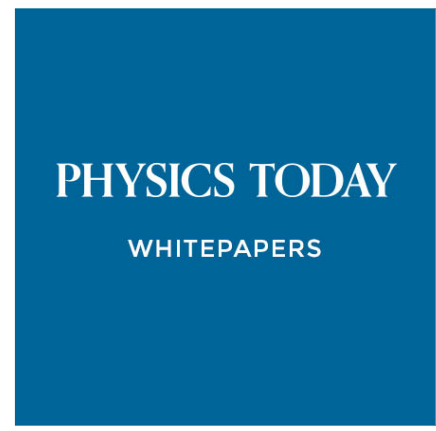




\title{
Polymer dynamics in bimodal polyethylene melts: A study with neutron spin echo spectroscopy and pulsed field gradient nuclear magnetic resonance
}

\author{
S. Rathgeber, ${ }^{\text {a) }}$ L. Willner, and D. Richter \\ Forschungszentrum Jülich, Institut für Festkörperforschung, 52425 Jülich, Germany \\ A. Brulet \\ Laboratoire Leon Brillouin, CE-Saclay, 91191 Gif Sur Yvette Cedex, France \\ B. Farago \\ Institute Laue-Langevin, BP 156, 38042 Grenoble Cedex 9, France \\ M. Appel ${ }^{\text {b) }}$ and G. Fleischer \\ Universität Leipzig, Institut für Experimentalphysik 1, Linnéstr. 5, 04103 Leipzig, Germany
}

(Received 28 December 1998; accepted 1 March 1999)

\begin{abstract}
We have investigated the dynamics of polymers in bimodal polyethylene (PE) melts in the transition region from Rouse- to reptationlike behavior by varying the mass fraction $\Phi_{t}$ of long tracer chains $\left(N \approx 3 N_{e}\right.$ or $\left.4 N_{e}\right)$ in a short-chain matrix $\left(N \approx N_{e}=\right.$ entanglement segment number) over the full concentration range. At short times (ns) the dynamic structure factor for single-chain relaxation was investigated by neutron-spin-echo (NSE) spectroscopy. To obtain information about the long-time (ms) dynamics the tracer diffusion coefficient $\left(D_{\mathrm{NMR}}\right)$ was measured by pulsed-field-gradient (PFG)-NMR. We discuss our NSE data within a mode analysis which includes the relaxation rates $W_{p}$ of the independent normal modes of the internal chain dynamics and the center-of-mass diffusion coefficient $D_{\mathrm{NSE}}$ as model parameters. Only modes exceeding the $\Phi_{t}$-dependent length of a single entanglement strand $N_{e}\left(\Phi_{t}\right)$ are found to be strongly hindered by topological constraints. The $D_{\mathrm{NSE}}$ are $\Phi_{t}$-independent and systematically faster than the strong concentration-dependent $D_{N M R}$, suggesting an effective time-dependent diffusion coefficient. The Hess model, which we have generalized for polydisperse melts, provides a time-dependent diffusion coefficient. Taking chain-end effects into account we get an excellent description of the NSE data. The mobility of the chain ends is much higher than the mobility of the inner segments resulting in an entanglement segment number which increases with decreasing tracer concentration. The concentration dependence of $N_{e}\left(\Phi_{t}\right)$, as obtained from the mode analysis and the Hess model, is in agreement with our calculation within a self-consistent modification of the model by Kavassalis and Noolandi for entanglement formation. (C) 1999 American Institute of Physics. [S0021-9606(99)50520-5]
\end{abstract}

\section{INTRODUCTION}

The understanding of polymer dynamics on a microscopic length- and time-scale is essential for predictions of the macroscopic properties of polymers such as viscoelasticity and diffusion which largely determine the possible applications and processing characteristics of these materials.

In this study we have investigated the dynamics of single chains by studying their coherent dynamic structure factor in the ns time range with neutron-spin-echo (NSE) spectroscopy, at length scales up to about $10 \mathrm{~nm}$. From complementary macroscopic measurements with pulsed-field-gradient (PFG) nuclear magnetic resonance (NMR), a method addressing diffusive motions in the $\mu \mathrm{m}$ range occurring on a ms time scale, center-of-mass diffusion coefficients were obtained.

In the limit of long chains and long times, the basic

\footnotetext{
${ }^{a)}$ Present address: National Institute of Standards and Technology, NIST Center for Neutron Research, 100 Bureau Dr., Stop 8562, Gaithersburg, Maryland 20899-8562; Electronic mail: silke.rathgeber@nist.gov

${ }^{b}$ Present address: Shell Exploration and Production Company, Bellaire Technology Center, 3737 Bellaire Blvd., Houston, Texas 77025.
}

concept of the reptation model of de Gennes (1967) (Refs. 1 and 2) and Doi and Edwards (1978) (Ref. 3) provides a good description of most macroscopic and microscopic features of dense polymeric systems, as does the Rouse model (1953) for the opposite limit of short chains and/or short times. The Rouse model, which is valid for length scales much longer than the statistical segment length $l$, describes the dynamics of noninteracting chains. In this model only one characteristic length scale, the end-to-end distance of the polymer, is relevant. The only parameter of the model is the Rouse rate $W_{R}$. Because of entanglements between polymers, the motion of longer chains is strongly hindered. Introducing a tubelike potential around a test chain the reptation incorporates the effect of topological constraints, caused by the surrounding medium, on the motion of a single chain. The favored motion is a reptationlike motion along the tube axis. The tube diameter $d$, identified with the distance between two entanglements or via $d=\sqrt{N_{e}} l$ with the number of segments between two entanglements, introduces, in comparison with the statistical segment length $l$ the end-to-end distance $R_{E}$ of the molecule, an intermediate length scale. The entanglement distance $d$ is a dynamic quantity. 
The aim of this study is to investigate the influence of topological constraints between chains on the chain dynamics in the transition region between Rouse- and reptationlike behavior where the tube picture of the reptation model does not hold any more. The Rouse to reptation transition region is probed by varying the fraction of longer tracer chains $\left(N_{t} \approx 3\right.$ or $\left.4 N_{e}\right)$ in a short-chain matrix $\left(N_{m} \approx N_{e}\right)$. We will show in Sec. IV B and Sec. IV C that the influence of the entanglement distance (tube diameter) on the chain dynamics is still observable even for the short chains investigated here.

Efforts have been made to develop theories that also cover the transition region between the short-time Rouse relaxation and the long-time creep motion of the reptation model. Two models addressing the long-chain limit are the Ronca model (1983) (Ref. 4) and the rubberlike model of des Cloizeaux (1993). ${ }^{5}$ Starting from the analogy between a rubber and a polymer melt, the des Cloizeaux model treats the entanglements as spatially fixed and allows Rouse relaxation between these temporary crosslinks. This approach limits the model to times significantly shorter than the Rouse time $\tau_{R}$ where the one-dimensional diffusive motion along the tube is the dominant relaxation mechanism. The theory of Ronca takes the viscoelastic properties of a polymer melt into account by introducing a memory function in the equation of motion of a single test chain. This model provides a phenomenological ansatz for the kernel of the memory function. Recently published NSE measurements on long polyethylene melts ${ }^{6}$ strongly question the validity of these theories and clearly favor the reptation model.

Two theories not limited to long chains are the model of Chatterjee and Loring (1994) (Ref. 7, binary blends see Ref. 8), and the theory formulated by Hess (1986). ${ }^{9-11}$ The stochastic model of Chatterjee and Loring describes the polymer dynamics as a combination of a local kink-jump motion and a slithering motion in a field of fluctuating obstacles whose relaxation are coupled to the slowest internal relaxation mode of the test chain. The predicted decay of the dynamic structure factor is too rapid when compared to experimental results. ${ }^{6,7}$

We wish to focus interest here on the microscopic model of Hess, which takes the excluded-volume interaction between polymer chains into account, resulting (as does the Ronca model) in a memory term in the equation of motion of a single test chain. In contrast to the Ronca model, a microscopic explanation for the kernel of the memory function is given. A comparison of the theoretical predictions of this theory with experimental NSE measurements on polyethylene melts with molecular weight in the transition region from Rouse- to reptationlike behavior shows excellent agreement between theory and experiment (Sec. IV C). In addition to the Rouse model, the Hess model employs two further parameters: the range of the excluded-volume interaction $q_{c}^{-1}$ and the critical segment number $N_{c}$. In a monomodal melt with $N \geqslant N_{c}$ the chain motion perpendicular to the chain contour freezes. This has been interpreted by Hess as a Rouse- to reptation transition. The basic predictions of the Hess model are an effective time-dependent center-of-mass diffusion coefficient and a two-step relaxation mechanism for internal relaxation modes (Sec. II B). We should also mention the mode coupling theory of Schweizer and Szamel (1989). ${ }^{12-14}$ This microscopic theory is based on an explicit nonlinear coupling of the collective density fluctuations of the polymeric fluid with the segmental density field of the probe polymer. The only tractable approximation-a renormalized Rouse theory ${ }^{14}$ - stands in contradiction to experimental results for macroscopic quantities such as diffusion coefficient and viscosity, as well as to the results obtained by Richter et al. ${ }^{15}$ with NSE. Unfortunately the authors only applied their model in a more general form to macroscopic quantities ${ }^{16,17}$ a calculation of the dynamic structure factor is still missing.

We conclude that for the present purpose, the Hess model appears to be the most promising approach to describe the polymer dynamics in the transition region between Rouse- and reptationlike behavior. In Sec. IV C we will show the excellent agreement between theory and NSE results. The model was originally formulated for monomodal systems. In Sec. II B we extend it to polydisperse systems.

In addition to a discussion of our data within the framework of the microscopic Hess model we have performed a mode analysis (introduced in Sec. II A) that gives us separate access to the independent normal modes of the chain, each describing a polymer motion on a different length scale. The model parameters are the center-of-mass diffusion coefficient $D$ and the mode-dependent relaxation rates $W(p)$.

The experimental part includes in Sec. III A a description of the synthesis, characterization of the polymers, an overview of the investigated samples and a short introduction to the complementary techniques of NSE spectroscopy, in Sec. III B, and PFG-NMR, in Sec. III C.

In Sec. IV A we discuss our results for the long-time center-of-mass diffusion coefficient which shows a strong dependence on the tracer concentration $\Phi_{t}$. This observation cannot be explained by an increase of the free volume with decreasing tracer fraction. Therefore, we apply the Hess model together with an ansatz for a concentration-dependent value of $N_{e}$. The NSE spectra are discussed in Sec. IV B in terms of the mode analysis. Modes describing chain motion on length scales exceeding the tracer concentrationdependent distance between two entanglements are found to be strongly hindered by topological constraints. The results for the center-of-mass diffusion coefficient are independent of concentration and systematically larger than the PFGNMR results. This observation strongly suggests an effective time-dependent center-of-mass diffusion coefficient.

The Hess model predicts a time-dependent diffusion coefficient and we show in Sec. IV C that taking chain-end effects into account enables us to achieve excellent description of the short-time dynamics (NSE) and the long-time dynamics.

In the low molecular weight range that we have investigated chain-end effects cannot be neglected. Because of the higher degree of freedom of the chain-end segments $N_{c}$ (e.g., $N_{e}$ ) depends on the tracer concentration. In Sec. IV D we calculate $N_{e}\left(\Phi_{t}\right)$ using a modification of the model for entanglement formation due to Kavassalis and Noolandi. ${ }^{18,19}$

The paper ends with the conclusions given in Sec. V. 


\section{THEORY}

\section{A. Mode analysis}

Under the assumption that topological constraints will change the relaxation spectrum of each mode differently but not their spatial structure (the chain remains Gaussian) the mode analysis parameterizes the effect of entanglements into length-scale-dependent relaxation rates.

The aim of this analysis is to investigate the influence of topological constraints on the individual modes of a relaxing chain. For this purpose we start from the fact that, independent of the complexity of the relaxation processes involved, the trajectory of a Gaussian chain can generally be decomposed into Fourier components $x_{p}$, the so-called Rouse modes, ${ }^{20}$

$$
x_{p}=\frac{1}{N} \sum_{n=1}^{N} r_{n}(t) \cos \left(\frac{p n \pi}{N}\right),
$$

where $N$ is the number of chain segments and $r_{n}$ are their time $(t)$-dependent position vectors. The mode number $p$ gives the number of knots of the Rouse modes along the chain. From a physical point of view, each of these independent normal modes $x_{p}$ describes a relaxation of the chain involving $N / p$ segments. $N / p$ is the number of segments between two knots and corresponds to a length scale of order $\sqrt{N l^{2} / p}$. Here $l$ is the length of a statistical segment which was determined ${ }^{21}$ to be $l^{2}=C_{\infty} l_{0}^{2}=13.76 \AA^{2}$ for PE melts at $T=509 \mathrm{~K}\left(l_{0}=\right.$ bond length $=1.53 \AA$ and $C_{\infty}=$ characteristic ratio $=5.87$ ).

The coherent dynamic structure factor $S(q, t)$ of a relaxing Gaussian chain can be expressed in terms of $x_{p}$. The result for $S(q, t)$ is a product of two contributions, ${ }^{20}$

$$
\begin{aligned}
S(q, t)= & \frac{1}{N} \exp \left(-\frac{q^{2}}{6}\left\langle\left[x_{0}(t)-x_{0}(0)\right]^{2}\right\rangle\right) \\
& \times\left\{\sum _ { m , n } ^ { N } \operatorname { e x p } \left[-\frac{q^{2} l^{2}}{6}|m-n|-\frac{4 R_{g}^{2} q^{2}}{\pi^{2}}\right.\right. \\
& \times \sum_{p=1}^{N} \frac{1}{p^{2}} \cos \left(\frac{p \pi m}{N}\right) \cos \left(\frac{p \pi n}{N}\right) \\
& \left.\left.\times\left(1-\left\langle x_{p}(t) x_{p}(0)\right\rangle\right)\right]\right\} .
\end{aligned}
$$

Here $q$ is the scattering vector, $R_{g}$ is the radius of gyration, and $x_{0}$ is the center-of-mass coordinate. The first term describes the diffusive motion of the center-of-mass in terms of the time correlation function of the mean square center-ofmass displacement $\left\langle\left[x_{0}(t)-x_{0}(0)\right]^{2}\right\rangle$. The second term, that contains the time correlation functions of the internal normal modes $\left\langle x_{p}(t) x_{p}(0)\right\rangle$, relates to the chain dynamics on length scales smaller than the end-to-end distance $R_{e}$ of the polymer.

To evaluate the temporal development of the system described by the correlation functions $\left\langle x_{p}(t) x_{p}(0)\right\rangle$, detailed considerations of the involved relaxation processes are necessary.
We start with the Rouse model ${ }^{22,20}$ which disregards excluded-volume and hydrodynamic interactions between chains. In this model the surrounding chains are assumed to provide only a heat bath (stochastic forces) and relaxation occurs under the influence of entropic restoring forces and segmental friction. Under this assumption, the time dependence of the normalized correlation functions are single exponential,

$\left\langle x_{p}(t) x_{p}(0)\right\rangle=\exp \left(-p^{2} / \tau_{R} t\right)$ with $\tau_{R}=\frac{\zeta N^{2}}{3 \pi^{2} k T}=\frac{N^{2}}{W \pi^{2}}$,

$\left\langle\left[x_{0}(t)-x_{0}(0)\right]^{2}\right\rangle=6 D_{R} t=\frac{k T}{N \zeta} t$.

$\zeta$ is the monomeric friction coefficient, $D_{R}$ the Rouse diffusion coefficient, $W_{R}$ the Rouse rate, and the Rouse time $\tau_{R}$ is the characteristic time of the model.

The validity of the Rouse model ${ }^{23}$ is limited to chains with molecular weights lower than the entanglement molecular weight and/or times shorter than the entanglement time $\tau_{e}$, where the entanglements have no influence on the chain dynamics. In both cases the influence of topological constraints on the chain motion can be neglected. From previous measurements $(T=509 \mathrm{~K})$ on high molecular weight PE samples, ${ }^{24,21}$ the entanglement molecular weight is known to be $M_{e} \approx 2000 \mathrm{~g} / \mathrm{mol}$, corresponding to a segment number between two entanglements of $N_{e} \approx 140$. The entanglement time $\tau_{e}$ is defined as the characteristic Rouse time of a chain with an end-to-end distance of the same value as the distance $d$ between two entanglements; $\tau_{e}$ was determined ${ }^{21}$ for PE at $509 \mathrm{~K}$ to be approximately $5 \mathrm{~ns}$. For chains above the entanglement molecular weight, such as those investigated here, no satisfactory description of the data over the entire time range is obtained in terms of the Rouse model. ${ }^{15}$

The mode analysis is a generalization of the Rouse model. From static form factor measurements we know that under the influence of entanglements the statistics of the chains stay Gaussian (see, for example, Ref. 25). The spatial structure of the modes should therefore remain of Rousetype. Unlike the Hess model which considers time-dependent friction, in the mode analysis we assume that the time dependence of the correlation functions $\left\langle x_{p}(t) x_{p}(0)\right\rangle$ is given by a single exponential decay but the characteristic relaxation time is, in contrast to the Rouse model, assumed to be a function of the mode number $p$,

$$
\tau_{R} \rightarrow \tau_{p}=\tau(p) \text { or } W_{R} \rightarrow W_{p}=W(p) .
$$

This parameterization allows the hindrance of the polymer dynamics arising from topological constraints to be of different strengths for modes corresponding to different length scales. In other words, the friction acting on the segments is now a function of the length scale of the segment dynamics, $\zeta \propto 1 / W_{p}$.

The ability of neutron scattering to observe the molecular motion on its natural time- and length $(1 / q)$-scale allows a separation of the contribution of different modes to $S(q, t)$ - different modes affect different $(q-t)$ regimes. Modes with low mode numbers and the translational diffu- 
sion contribution $(p=0)$ describe the long-time and large length-scale behavior (at small $q$-values) of $S(q, t)$. Higher modes are necessary to give a satisfactory description of the short-time and small length-scale region. ${ }^{15}$

Practical fitting involves the following parameters: the translational diffusion coefficient $D$, the relaxation rates for the first four (or three) modes and another rate for all higher modes $(p>5$ or 4$): W(p)=W(4$ or 5$)$.

\section{B. Generalized Rouse model by Hess}

The intention of the generalized Rouse model formulated by Hess ${ }^{11,10,9}$ is to give a microscopic description of the relaxation and diffusion processes in dense polymeric systems. We present here a generalization of this theory to polydisperse systems. The basic concept is the connectivity and impenetrability of the polymers. In contrast to simple liquids the information about a collision between two segments is transmitted along the chain and may affect major parts of a molecule. In the Rouse model the characteristic times of these collisions are assumed to be much shorter than the characteristic relaxation times of the chains. Including the excluded-volume interaction in the Hess model, both time scales become of the same order, and the collisions are no longer simply local events in time.

The starting point is the Hamiltonian of continuous elastic chains with excluded-volume interaction, the Edwards-Hamiltonian ${ }^{20}$

$$
\begin{aligned}
H_{E}= & \frac{3 k T}{2 l^{2}} \sum_{i=1}^{n} \int_{0}^{N_{i}} d s_{i}\left(\frac{\partial \mathbf{r}\left(s_{i}\right)}{\partial s_{i}}\right)^{2}+\frac{v_{0} k T}{2} \\
& \times \sum_{i, i^{\prime}=1}^{n} \int_{0}^{N_{i}} d s_{i} \int_{0}^{N_{i^{\prime}}} d s_{i^{\prime}} \delta\left(\mathbf{r}\left(s_{i}\right)-\mathbf{r}\left(s_{i^{\prime}}\right)\right) .
\end{aligned}
$$

The first term considers the elastic coupling of the segments in a given chain (Rouse term) and the second term contains the excluded-volume interaction between two segments. Here $v_{0}$ is the excluded-volume parameter, $n$ is the number of chains, $N_{i}$ the number of segments of an individual chain, $s_{i}=1 \ldots N_{i}$ the segment index, $i=1 \ldots n$ numbers the chains, and $k$ denotes the Boltzmann constant.

For the motion of the segments of a test chain with index $t$ and $N_{t}$ segments via the Mori-Zwanzig projection formalism, ${ }^{26}$ generalized Rouse equations are obtained,

$$
\begin{aligned}
\zeta \dot{\mathbf{r}}(s, t) & +\frac{3 k T}{l^{2}} \sum_{s^{\prime}=1}^{N_{t}} A_{s, s^{\prime}} \mathbf{r}\left(s^{\prime}, t\right) \\
& +\sum_{s^{\prime}=1}^{N_{t}} \int_{0}^{t} d t^{\prime} \Delta \zeta_{s, s^{\prime}}\left(t-t^{\prime}\right) \dot{\mathbf{r}}\left(s, t^{\prime}\right)=\mathbf{f}(s, t) .
\end{aligned}
$$

Here $\mathbf{f}(s, t)$ are the random forces acting from the heat bath on the segments of the test chain and $A_{s, s^{\prime}}$ is the nearest neighbor interaction matrix. ${ }^{22}$ In comparison to the Rouse model this equation contains an additional term, a memory function where the entanglement friction matrix $\Delta \zeta_{s, s^{\prime}}$ appears as kernel. This term takes the viscoelastic properties of the medium into account. In the related Ronca model the kernel of the memory function follows from semiphenom- enological considerations. Here, as a direct result of the projection operator method, $\Delta \zeta_{s, s^{\prime}}$ can be expressed as the autocorrelation function of the total excluded-volume forces $\mathbf{F}_{\mathrm{ev}}$ acting on the test chain,

$$
\Delta \zeta_{s, s^{\prime}}(t)=\frac{1}{3 k T N_{t}}\left\langle\mathbf{F}_{\mathrm{ev}}(s, t) \mathbf{F}_{\mathrm{ev}}\left(s^{\prime}, 0\right)\right\rangle .
$$

In Eq. (6) the motion of each segment of the test chain is coupled via the entanglement friction matrix with the motion of all other segments of the system. Starting from the Fourier representation of the excluded-volume forces, the entanglement friction function is a dynamic four-point correlation function which cannot be calculated rigorously. A decoupling of the equations of motion can be achieved by a factorization in a lowest order mode-coupling approximation. This means physically that (1) the information on a specific collision is subsequently transmitted along a chain and therefore additional collisions at intermediate times are neglected and (2) disregarding more complicated correlations between many chains the main contribution to the relaxation of $\Delta \zeta_{s, s^{\prime}}$ originates from the single segment propagators $G_{i}(q, t)$. Under this assumption the entanglement friction function can now be written as

$\Delta \zeta(t)=\frac{\varphi}{3 V} \frac{v_{0}}{(2 \pi)^{3}} \int^{|q|=q_{c}} d^{3} q q^{2}\left(G_{t}(q, t) \sum_{i \neq t} N_{i} G_{i}(q, t)\right)$,

where $V$ is the volume of the system. The index $t$ denotes the test chain while the index $i$ relates to the other chains with a degree of polymerization $N_{i}$. The integration is limited to the range $q_{c}^{-1}$ of the true excluded-volume interaction potential and $\varphi$ is the mean excluded-volume energy between two arbitrary segments $s_{i}$ and $s_{i}$ in the discrete chain model. Under the assumption that all segments are statistically equivalent, and neglecting chain-end effects, in the longchain limit $\varphi$ can be written as

$$
\varphi=V v_{0} k T\left\langle\delta\left(\mathbf{r}\left(s_{i}\right)-\mathbf{r}\left(s_{i^{\prime}}\right)\right)\right\rangle .
$$

One important feature in Eq. (8) is the occurrence of the single-chain propagators in second order. Consequently, not only the motion of the test chain but also the dynamics of the surrounding chains contribute to the relaxation of the entanglement friction function. This means that constraintrelease mechanisms similar to the des Cloiseaux concept of double reptation ${ }^{27-29}$ are automatically considered. The single-chain propagators in Gaussian approximation are

$$
G_{i}(q, t)=\exp \left(-q^{2} W_{i}(t)\right),
$$

where $6 \cdot W_{i}(t)$ is the mean square displacement of a segment. Under the assumption that chain-end effects and curvilinear density fluctuations have to be considered only as small perturbations, the curvilinear and lateral diffusive contribution to the mean square center-of-mass displacement decouples and $W_{i}(t)$ can be written as

$$
W_{i}(t)=D_{i}^{\|} t+D_{i}^{\perp} t+W_{i}^{\mathrm{int}}(t) .
$$

Here $D_{i}^{\|}$and $D_{i}^{\perp}$ are the long-time center-of-mass diffusion coefficients parallel and perpendicular to the chain contour and $W_{i}^{\text {int }}(t)$ are the internal chain displacements. Assum- 
ingthat the main mechanism for the dissolution of an entanglement is the diffusive sliding of two chains which is strictly true only for long chains, the prevailing relaxation of the entanglement friction function takes place on the time scale of center-of-mass diffusion. Therefore the contribution of internal displacements $W_{i}^{\text {int }}$ is negligible. Introducing the characteristic times

$$
\tau_{t i}=\left[\left(D_{t}^{\|}+D_{t}^{\perp}+D_{i}^{\|}+D_{i}^{\perp}\right) q_{c}^{2}\right]^{-1},
$$

the entanglement friction function in Eq. (8) simplifies to

$$
\Delta \zeta(t)=\frac{\varphi}{V} q_{c}^{2} \int_{0}^{1} d y y^{4} \sum_{i \neq t} N_{i} \exp \left(-t y^{2} / \tau_{t i}\right) .
$$

Partial integration then leads to an analytical result for $\Delta \zeta(t)$

$$
\begin{aligned}
\Delta \zeta(t)= & \frac{\varphi}{V} q_{c}^{2} \sum_{i \neq t} N_{i}\left[\exp \left(-\frac{t}{\tau_{t i}}\right)\left(-\frac{\tau_{t i}}{2 t}-\frac{\tau_{t i}^{2}}{t^{2}}\right)\right. \\
& \left.+\frac{3 \sqrt{\pi}}{8}\left(\frac{\tau_{t i}}{t}\right)^{5 / 2} \operatorname{erf}\left(\sqrt{\frac{t}{\tau_{t i}}}\right)\right] .
\end{aligned}
$$

From the approximately translational invariance of the interaction potential $D_{j}^{\|}$is derived to be the diffusion coefficient of a one-dimensional Rouse chain, and a self-consistent set of algebraic equations for $D_{i}^{\perp}$ can be obtained,

$$
\begin{aligned}
& D_{i}^{\|}=\frac{1}{3} \frac{k T}{N_{i} \zeta}=\frac{1}{3} D_{i}^{R}, \\
& D_{i}^{\perp}=\left(\frac{1}{\frac{2}{3} D_{i}^{R}}+\frac{1}{2} \frac{1}{k T} \frac{\varphi}{V} \sum_{i^{\prime} \neq i} \frac{N_{i^{\prime}} N_{i}}{D_{i^{\prime}}^{\perp}+D_{i}^{\perp}}\right)^{-1} .
\end{aligned}
$$

For the overall center-of-mass diffusion coefficient $D_{i}$ in the limit of long times, the following expression results:

$$
D_{i}=\left(\frac{1}{D_{i}^{R}}+\frac{1}{3} \frac{1}{k T} \frac{\varphi}{V} \sum_{i^{\prime} \neq i} \frac{N_{i^{\prime}} N_{i}}{D_{i^{\prime}}^{\perp}+D_{i}^{\perp}+D_{i^{\prime}}^{\|}+D_{i}^{\|}}\right)^{-1} .
$$

In general, a solution of the Eq. (16) will only be obtained numerically, but in the special case of monomodal melts an analytical solution for $D^{\perp}$ exists,

$$
\begin{aligned}
D^{\perp} & =\frac{2}{3}\left(\frac{1}{D_{R}}+\frac{1}{6} \frac{N}{k T} \frac{\varphi}{V} \frac{1}{D^{\perp}}\right)^{-1} \\
& = \begin{cases}\frac{2}{3} D_{R}\left(1-\frac{N}{N_{c}}\right) & \text { for } N \leqslant N_{c} \\
0 & \text { for } N \geqslant N_{c}\end{cases}
\end{aligned}
$$

The fact that the lateral motion above a certain critical segment number $N_{c}$ freezes and only curvilinear diffusion is allowed has been interpreted by Hess as a Rouse to reptation transition. In contrast to the reptation model, where the reptative motion was enforced by introducing a tubelike potential around a test chain, here the freezing of the lateral motion is discussed as a consequence of the interaction potential against curvilinear displacements. The energy of interaction between chains is proportional to the number of interchain contacts. This number does not change if the polymers move along their contour lines. It should be mentioned that this strictly holds only in the long-chain limit-the finite length is considered by taking chain-end effects and curvilinear density fluctuations as small perturbations into account. The critical segment number $N_{c}$ is directly connected to the mean excluded-volume interaction energy between two segments $\varphi$,

$$
N_{c}=\frac{4 k T v_{s}}{\varphi}
$$

with $v_{s}=$ segment volume. From measurements performed at $T=509 \mathrm{~K}$ on monomodal PE melts with different molecular weights, ${ }^{15} N_{c}$ is found to be $\approx 150$ and therefore it has been identified with the entanglement segment number $N_{e} \approx 140$ determined for long $\mathrm{PE}$ chains at the same temperature.

Our interest lies in bimodal melts where the segment number $N_{t}$ of the labeled tracer polymers $\left(N_{t}=366\right.$ or 583$)$ is larger than the critical segment number. It can be shown that for all concentrations considered the lateral diffusion coefficient $D_{t}^{\perp}$ vanishes. For the matrix molecules with a segment number $N_{m}=$ (126 or 130) smaller than $N_{c}$ the following expressions are obtained:

$$
D_{m}^{\perp}=\left\{\begin{array}{ll}
\frac{2}{3} D_{m}^{R}\left(1-\frac{N_{m}}{N_{c}}\left(\Phi_{t}+1\right)\right) & \text { for } \Phi_{t} \leqslant \frac{N_{c}}{N_{m}}-1 \\
0 & \text { for } \Phi_{t} \geqslant \frac{N_{c}}{N_{m}}-1
\end{array} .\right.
$$

Finally, substituting this expression into Eq. (17) the overall center-of-mass diffusion coefficient $D_{t}$ of the tracer is determined to be

$$
D_{t}=\left\{\begin{array}{ll}
D_{t}^{R}\left(1+2 \Phi_{t} \frac{N_{t}}{N_{c}}+\frac{4\left(1-\Phi_{t}\right) N_{t} N_{m}}{N_{c}\left(N_{m}+3 N_{t}\right)-2 N_{t} N_{m}\left(1+\Phi_{t}\right)}\right)^{-1} & \text { for } \Phi_{t} \leqslant \frac{N_{c}}{N_{m}}-1 \\
D_{t}^{R}\left(1+2 \Phi_{t} \frac{N_{t}}{N_{c}}+\frac{4\left(1-\Phi_{t}\right) N_{t} N_{m}}{N_{c}\left(N_{t}+N_{m}\right)}\right)^{-1} & \text { for } \Phi_{t} \geqslant \frac{N_{c}}{N_{m}}-1
\end{array} .\right.
$$


With the determination of $D^{\|}$and $D^{\perp}$ for each component the entanglement friction function in Eq. (13) is defined and the generalized Rouse equations [Eq. (6)] can now be solved by Fourier transformation (described in detail in Refs. 11 and 15). This method gives the frequency-dependent correlation functions $\left\langle x_{p}(\omega) x_{p^{\prime}}\left(\omega^{\prime}\right)\right\rangle$,

$p>0:\left\langle x_{p}(\omega) x_{p}\left(\omega^{\prime}\right)\right\rangle$

$$
=D_{t}^{R} \frac{\left[1+\sqrt{(2 \pi)} \frac{\Delta \zeta(\omega)}{\zeta}\right] \delta\left(\omega+\omega^{\prime}\right)}{\text { denominator }},
$$

$p=0:\left\langle x_{0}(\omega) x_{0}\left(\omega^{\prime}\right)\right\rangle=2 D_{t}^{R} \frac{1}{\omega^{2}} \frac{\delta\left(\omega+\omega^{\prime}\right)}{1+\sqrt{2 \pi}} \frac{\Delta \zeta(-\omega)}{\zeta}$,

where the denominator is given by

$$
\begin{aligned}
\text { denominator }= & \left(\frac{p^{4}}{\tau_{R}}\right)^{2}-\omega \sqrt{8 \pi} \frac{p^{4}}{\tau_{R}} \operatorname{Im}\left(\frac{\Delta \zeta(\omega)}{\zeta}\right) \\
& +\omega\left[1+\sqrt{8 \pi} \operatorname{Re}\left(\frac{\Delta \zeta(\omega)}{\zeta}\right)+2 \pi\left|\frac{\Delta \zeta(\omega)}{\zeta}\right|^{2}\right],
\end{aligned}
$$

and for the Fourier transform of the entanglement friction function one obtains

$$
\begin{aligned}
\Delta \zeta(\omega)= & \frac{\varphi}{V} q_{c}^{2} \sum_{i \neq t} N_{i}\left[1-\frac{1}{3} \omega \tau_{t i}-\frac{3}{\sqrt{2}}(i-1)\right. \\
& \left.\times\left(\omega \tau_{t i}\right)^{3 / 2} \arctan \left(\frac{i-1}{\sqrt{2} \omega \tau_{t i}}\right)\right] .
\end{aligned}
$$

For the determination of the dynamic structure factor of a Gaussian chain the time-dependent correlation functions $\left\langle x_{p}(t) x_{p^{\prime}}\left(t^{\prime}\right)\right\rangle$ are necessary. The back transformation can only be performed numerically (e.g., using the fast-Fourier routine C06FCF of the NAG library). After insertion into Eq.

(2) a numerical expression for $S(q, t)$ results.

Finally, in addition to the only parameter of the Rouse model-the Rouse rate $W_{R}$ - the Hess theory involves two further parameters; the range of the excluded-volume interaction potential $q_{c}^{-1}$ and the critical segment number $N_{c}$. The Rouse rate enters into the model through the Rouse diffusion coefficient.

To compare our results with those obtained in previous publications we want to introduce another quantity

$$
\frac{\Delta \zeta(t=0)}{\zeta}=\frac{4}{15} W_{R} l^{2} \frac{1}{q_{c}^{-2} N_{c}},
$$

that measures the strength of the entanglement friction relative to the monomeric friction. The entanglement friction increases with decreasing range of the excluded-volume interaction $q_{c}^{-1}$ and decreasing critical segment number $N_{c}$.

As a special feature the Hess model provides a timedependent diffusion coefficient which in the short-time limit equals the Rouse value $D_{R}$ and for long times is given by Eq. (21). A representative time dependence is shown in Fig. 1, where the mean-square center-of-mass displacement $\left\langle\left[\mathbf{r}_{0}(t)-\mathbf{r}_{0}(0)\right]^{2}\right\rangle=6 D(t) \times t$ is plotted vs time for one of the investigated systems $\left(87.5 \% \quad N_{t}=552, N_{m}=130\right)$. The model parameters are taken from an analysis of the NSE spectra within the Hess model (see Sec. IV C). Obviously the crossover from short- to long-time behavior lies in the experimental time range $(0.1 \mathrm{~ns} \leqslant t \leqslant 20 \mathrm{~ns})$ of the NSEspectrometer employed. The differences in the long- and short-time values of the center-of-mass diffusion coefficients are important for the comparison of the PFG-NMR data with the results of the fit of the NSE data within the mode analysis. Notice that an effective time-dependent diffusion coefficient is also obtained within the framework of the reptation model for times longer than the entanglement time $\tau_{e}$ and shorter than the reptation time $\tau_{d}$-the time when the chain has left its original tube.

Evaluating the time-dependent correlation functions $\left\langle x_{p}(t) x_{p}(0)\right\rangle$ from the Hess theory, we can now present the effect of the topological constraints on the temporal development of the correlation functions. Figure 2 displays the results for the systems $\left(N_{t}=365, N_{m}=126\right)$ at low $\left(\Phi_{t}\right.$ $=10 \%)$ and high $\left(\Phi_{t}=87.5 \%\right)$ tracer concentrations. The time-correlation functions of the normal modes are plotted double-logarithmically as a function of time including a $1 / p^{2}$ prefactor to separate the different curves. Whereas for low tracer concentrations the first modes show significant relaxation within the experimental time window of the NSE experiment, increasing $\Phi_{t}$ causes a strong slowing down of these modes. At high tracer fractions, the relaxation of the dynamic structure factor is dominated by the higher modes which exhibit a two-step relaxation in contrast to the singleexponential relaxation of the Rouse model. After an initial decay to a mode-dependent plateau value final relaxation takes place at long times.

\section{EXPERIMENT}

\section{A. Sample preparation and characterization}

The polyethylene samples were prepared via the anionic route. (1) The polybutadienes $(\mathrm{PBd})$ were first synthesized

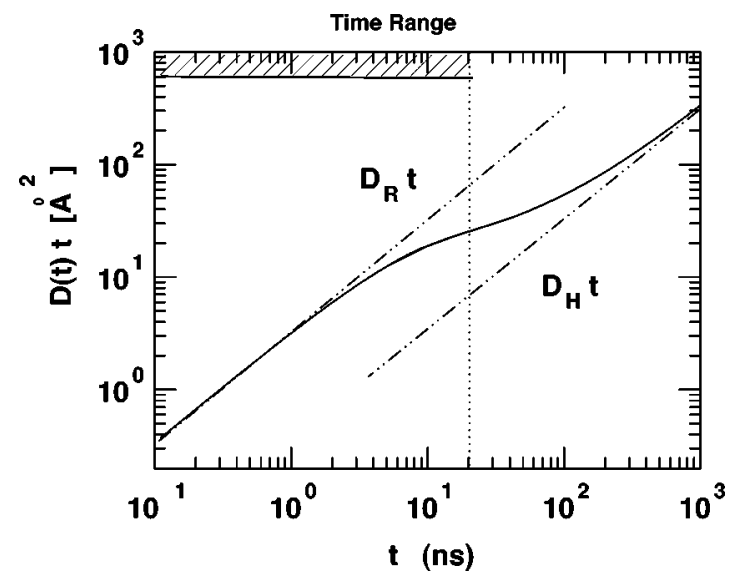

FIG. 1. Mean-square center-of-mass displacement $\left\langle\left[\mathbf{r}_{0}(t)-\mathbf{r}_{0}(0)\right]^{2}\right\rangle$ $=6 D(t) \times t$ of the tracer molecules as a function of time for the system ( $\left.87.5 \% N_{t}=583, N_{m}=130\right)$, using the parameters obtained from a fit within the Hess model. The broken lines show the short-time $\left(D_{t}^{R} t\right)$ behavior expected from Rouse theory and long-time behavior $\left(D_{t}^{H} t\right)$ as calculated within the Hess theory [Eq. (21)]. The time range of the NSE experiment is marked by the shaded area. 


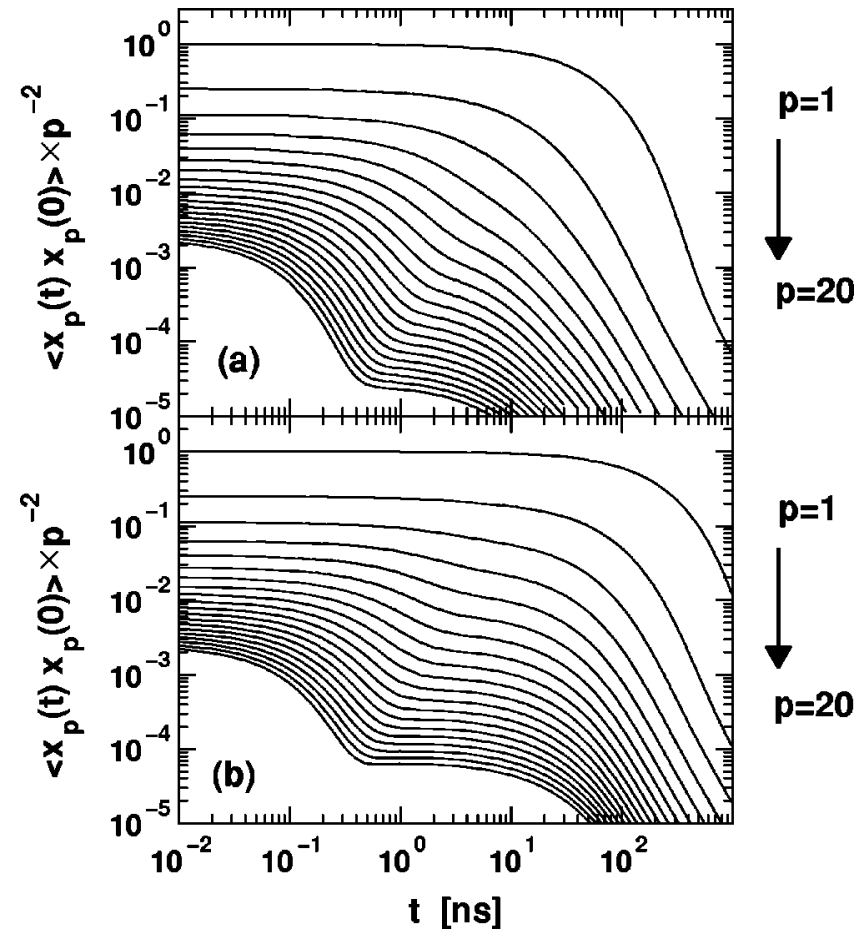

FIG. 2. Correlation functions $\left\langle x_{p}(t) x_{p}(0)\right\rangle / p^{2}$ calculated within the Hess theory vs time for the system $\left(N_{t}=365, N_{m}=126\right)$ at low (a) $\Phi_{t}=10 \%$ and high (b) $\Phi_{t}=87.5 \%$ tracer concentration. The parameters $N_{c}\left(\Phi_{t}\right)$ and $q_{c}^{-1}$ are obtained from a fit of the NSE spectra within the Hess theory (see Table $\mathrm{V})$. The prefactor $1 / p^{2}$ achieves a separation of the curves.

via standard anionic polymerization techniques using sec- or tert-butyllithium (purified by sublimation) as initiator. The deuterated monomers were obtained from Cambridge Isotopes. (2) Polyethylene was then obtained by saturation of PBd precursor polymers. The saturation of the polydienes by hydrogen or deuterium followed the procedure of Gelsen and Bates $^{30}$ using palladium on barium sulfate as catalyst. NMR analysis confirmed saturation levels of $>99.9 \%$. The saturation process generates a crystalline poly(ethylene-1-butene) copolymer, which is designated as PEB-2 and essentially resembles polyethylene. In the context of this paper we denote PEB-2 as polyethylene (PE). The integer denotes the approximate number of ethyl branches per 100 back- bone atoms - the exact ratio is $1.7 / 100$. Size-exclusion chromatography, vapor-pressure osmometry and/or NMR were performed to measure the molecular weight and heterogeneity indices of the precursor polymers; the values were corrected for saturation. The results are summarized in Table I.

The samples contained long tracer molecules $\left(N_{t}=366\right.$ or 552) in a short-chain matrix $\left(N_{m}=126\right.$ or 130). In the experiment the volume fraction $\Phi_{t}$ of the tracer molecules was changed over the full concentration range, keeping the labeled (protonated) fraction at $10 \%$. Table II gives an overview of the investigated samples.

\section{B. Neutron-spin-echo (NSE) spectroscopy}

The neutron scattering experiments were carried out at the "MESS" NSE spectrometer at the Orphee reactor in Saclay and at the NSE spectrometer at the Dido reactor in Jülich. In a NSE experiment the neutron polarization $P(\theta, H)$ is measured as a function of the external guide field $H$ for different scattering angles $\theta .{ }^{31,32}$ For quasielastic scattering $P(\theta, H)$ directly gives the normalized dynamic structure factor $S(q, t) / S(q, 0)$, where $q=(4 \pi / \lambda) \sin (\theta / 2)$ is the momentum transfer and $t$ is the time, which is proportional to $H$ and to the cube of the neutron wavelength $\lambda^{3}$. The scattering contrast is caused by differences between the scattering lengths of the deuterated matrix and the protonated tracer molecules. From a sample containing a mixture of fully protonated and fully deuterated polymers the dynamic structure factor of a single-chain or the intrachain correlation function is obtained.

By minimizing the incoherent background-90\% of the sample was deuterated-the NSE experiment measures directly the intrachain pair correlation function. The incoherent background of the protons and the scattering from the niobium cells can be neglected. The broad inelastic spectrum from the deuterated matrix [approximately $S(q, t) \propto \delta(t)$ in the time domain] causes a $q$-dependent shift of the NSE signal to smaller values. Because the signal from the matrix does not show an echo the appropriate background correction is taken into account by dividing the NSE signal from the sample with

TABLE I. Molecular weights $M_{w}^{\mathrm{PBd}}$ and heterogeneity indices $\left(M_{w} / M_{n}\right)^{\mathrm{PBd}}$ of the polybutadiene precursor polymers and the values corrected for saturation: $M_{w}^{\mathrm{PEB}-2}=m_{\mathrm{PEB}} / m_{\mathrm{PBd}} \times M_{w}^{\mathrm{PBd}} ; m_{\mathrm{PEB}-2}$ and $m_{\mathrm{PBd}}$ are the monomer weights of polyethylene and polybutadiene respectively.

\begin{tabular}{|c|c|c|c|c|c|}
\hline System & $\begin{array}{l}\text { Precursor } \\
\text { polymer }\end{array}$ & $\begin{array}{c}M_{w} \times 10^{-3} \\
(\mathrm{~g} / \mathrm{mol})\end{array}$ & $M_{w} / M_{n}$ & $\begin{array}{l}\text { Saturated } \\
\text { polymer }\end{array}$ & $\begin{array}{c}M_{w} \times 10^{-3} \\
(\mathrm{~g} / \mathrm{mol})\end{array}$ \\
\hline \multirow[t]{3}{*}{$\left(N_{t}=365, N_{m}=126\right)$} & PBd- $d_{6}$ & $2.1^{\mathrm{a}}$ & $1.07^{\mathrm{a}}$ & PEB-2- $d_{8}$ & 2.2 \\
\hline & & $5.5^{\mathrm{a}}$ & $1.03^{\mathrm{a}}$ & & 5.8 \\
\hline & PB- $h_{6}$ & $5.5^{\mathrm{a}, \mathrm{c}}$ & $1.03^{\mathrm{a}}$ & PEB-2- $h_{8}$ & 5.7 \\
\hline \multirow[t]{3}{*}{$\left(N_{t}=365, N_{m}=126\right)$} & PBd- $d_{6}$ & $2.1^{\mathrm{a}, \mathrm{b}}$ & $1.05^{\mathrm{a}}$ & PEB-2- $d_{8}$ & 2.2 \\
\hline & & $9.2^{\mathrm{a}, \mathrm{b}}$ & $1.02^{\mathrm{a}}$ & & 9.8 \\
\hline & PBd- $h_{6}$ & $8.2^{\mathrm{a}, \mathrm{b}}$ & $1.02^{\mathrm{a}}$ & PEB-2- $h_{8}$ & 8.5 \\
\hline
\end{tabular}

Characterization methods:

${ }^{a}$ Size-exclusion chromatography.

${ }^{b}$ Vapor-pressure osmometry.

${ }^{\mathrm{c}} \mathrm{NMR}$. 
TABLE II. Summary of the investigated bimodal melts for both investigation methods (a) NSE and (b) PFG-NMR. In all samples $10 \%$ of the tracers were protonated and $\left(\phi_{t}-10 \%\right)$ deuterated. The matrix was completely deuterated.

\begin{tabular}{ccccccccccc}
\hline \hline \multirow{2}{*}{$\begin{array}{c}\text { System } \\
{\left[\Phi_{t} N_{t},\left(1-\Phi_{t}\right) N_{m}\right]}\end{array}$} & $\begin{array}{c}\text { Investigation } \\
\text { method }\end{array}$ & 10 & 12.5 & 25 & 37.5 & 50 & 62.6 & 75 & 87.5 & 100 \\
\cline { 3 - 10 }$\left(N_{t}=365, N_{m}=126\right)$ & NSE & $\sqrt{ }$ & & $\sqrt{ }$ & & $\sqrt{ }$ & $\sqrt{ }$ & $\sqrt{ }$ & $\sqrt{ }$ & \\
& PFG-NMR & $\sqrt{ }$ & $\sqrt{ }$ & $\sqrt{ }$ & $\sqrt{ }$ & $\sqrt{ }$ & $\sqrt{ }$ & $\sqrt{ }$ & $\sqrt{ }$ & $\sqrt{ }$ \\
$\left(N_{t}=583, N_{m}=130\right)$ & NSE & $\sqrt{ }$ & & $\sqrt{ }$ & & $\sqrt{ }$ & $\sqrt{ }$ & $\sqrt{ }$ & $\sqrt{ }$ & \\
& PFG-NMR & $\sqrt{ }$ & & $\sqrt{ }$ & & $\sqrt{ }$ & $\sqrt{ }$ & $\sqrt{ }$ & $\sqrt{ }$ \\
\hline \hline
\end{tabular}

$$
b g=1-\frac{T^{s}}{T^{m}} \frac{\tau^{s}}{\tau^{m}} \frac{d^{s}}{d^{m}} \frac{S(q, 0)^{m}}{S(q, 0)^{s}},
$$

where the index $s$ stands for the sample and $m$ for the deuterated matrix, $T$ is the transmission, $d$ the sample thickness, and $\tau$ the measuring time. The investigated $q$-values were $q=(0.036,0.055,0.077,0.115$, and 0.155$) \AA^{-1}$ and with a neutron wavelength of $\lambda=6 \AA$, times up to $t_{\max }=18 \mathrm{~ns}$ were reached in Saclay; in Jülich we used a wavelength of $\lambda$ $=8 \AA$ and reached $t_{\max }=25 \mathrm{~ns}$. To relate the experimental results to previous measurements on monomodal polyethylene melts ${ }^{15}$ a temperature of $T=509 \mathrm{~K}$ was chosen. The samples were heated to this temperature in a vacuum furnace with a temperature stability of $\Delta T=1{ }^{\circ} \mathrm{C}$. To avoid degradation of the polymers by oxygen we used niobium containers sealed in an argon atmosphere. cells is The instrumental resolution function was determined using a lamellar graphite standard (Saclay) or a $\mathrm{MgO}$ powder (Jülich), measured at room temperature.

\section{Pulsed field gradient NMR spectroscopy (PFG-NMR)}

The PFG-NMR measurements were performed at the Faculty of Physics and Geosciences of the University of Leipzig. Two spectrometers were used, the FEGRIS 60 and the FEGRIS 400 with proton resonance frequencies of $60 \mathrm{MHz}$ and $400 \mathrm{MHz}$, respectively. Applying two field gradient pulses of duration $\delta$, magnitude $g$ and separation time $t$ the spin-echo signal of a PFG-NMR experiment is attenuated if the spins (protons) have changed their positions during the diffusion time $t .{ }^{33}$ The PFG-NMR is a generalized scattering experiment, ${ }^{34,35}$ and the spin-echo attenuation is identical to the intermediate incoherent scattering function obtained in a neutron scattering experiment. For a Gaussian chain in the diffusion limit the PFG-NMR signal yields $S_{\text {inc }}(q, t)=\exp \left(-q^{2} D t\right)$, where $D$ is the self-diffusion coefficient and $q=\gamma \delta g$ is the generalized scattering vector; $\gamma$ denotes the gyromagnetic ratio of the proton, which is the spin under investigation.

In the experiment, $g$ values up to $25 \mathrm{~T} / \mathrm{m}$ and $\delta$ values up to $2 \mathrm{~ms}$ were used. Therefore, the minimum displacement detectable with PFG-NMR (which is about $1 / q_{\max }$ ) is 100 $\mathrm{nm}$. Consequently, this method measures the (long-time) self-diffusion of the center-of-mass. The diffusion time $t$, which in a PFG-NMR experiment ranges from a few ms up to $1 \mathrm{~s}$, was chosen in our experiment, depending on the value of the self-diffusion coefficient of the sample, to be between
$10 \mathrm{~ms}$ and $100 \mathrm{~ms}$. The measurements were performed under inert conditions at a temperature of $453 \mathrm{~K}$ and, for a few samples, also at $433 \mathrm{~K}$ and $473 \mathrm{~K}$. The latter value is the highest temperature the NMR spectrometer could achieve. The experimental error of the absolute values of the selfdiffusion coefficients lies in the range of $5 \%-10 \%$. We have extrapolated the data to the temperature of the NSE experiment $(T=509 \mathrm{~K})$ via the temperature dependence (VogelFulcher-Thamann relationship) of the monomeric friction coefficient extensively investigated by Pearson et al. ${ }^{36,37}$ (see the Appendix). Including the extrapolated values the PFGNMR results are summarized in Table III.

\section{RESULTS AND DISCUSSION}

The aim of our measurements was to investigate the onset of the influence of topological constraints on the intrachain dynamics by increasing the fraction of long tracer chains $\left(N_{t}=365\right.$ or 583) in a short-chain matrix $\left(N_{m}=126\right.$ or 130). From previous measurements on monomodal melts it is known that to a good approximation the matrix chains undergo Rouse dynamics, whereas the motion of the tracer molecules is strongly hindered by topological constraints. In the experiment the dynamics of the tracers was investigated.

\section{A. PFG-NMR}

The PFG-NMR results for the long-time center-of-mass diffusion coefficients $D_{\mathrm{NMR}}$ are displayed in Fig. 3, extrapolated to the temperature $T=509 \mathrm{~K}$ of the NSE experiment; Table III summarizes the measured and extrapolated results. The value for the system $\left(N_{t}=583, N_{m}=130\right)$ at $\Phi_{t}=100 \%$ is calculated from the results of Pearson et al. ${ }^{36,37}$ (see Appendix). Obviously the tracer diffusion coefficients $D_{\mathrm{NMR}}$ show a strong concentration dependence, increasing as the fraction of long tracer molecules decreases. This increase of about a factor of 5 in the investigated concentration range cannot be explained by an increase in the free volume with decreasing tracer concentration. As seen in the Appendix the change in the free volume, which expresses itself in the molecular weight and concentration dependence of the monomeric friction coefficient, is of the order of $20 \%$ over the full concentration range.

The solid lines in Fig. 3 show the result of a first qualitative description of the PFG-NMR data with the reptation diffusion coefficient ${ }^{20}$

$$
D_{\text {rep }}=\frac{1}{3} D_{t}^{R} \frac{N_{e}}{N_{t}}
$$


TABLE III. Diffusion coefficients measured with PFG-NMR and the to $T$ $=509 \mathrm{~K}$ extrapolated values for both systems.

\begin{tabular}{|c|c|c|c|c|c|c|}
\hline \multirow{2}{*}{$\begin{array}{l}\text { System: } \\
\text { Temp (K): } \\
\Phi_{t}(\%)\end{array}$} & \multicolumn{4}{|c|}{$\left(N_{t}=365, N_{m}=126\right)$} & \multicolumn{2}{|c|}{$\left(N_{t}=583, N_{m}=130\right)$} \\
\hline & 433 & $\begin{array}{l}453 \\
D\left(10^{-11}\right.\end{array}$ & $\begin{array}{c}473 \\
\left.\mathrm{~m}^{2} / \mathrm{s}\right)\end{array}$ & 509 & $\begin{array}{l}453 \\
D\left(10^{-11}\right.\end{array}$ & $\begin{array}{c}509 \\
\left.\mathrm{~m}^{2} / \mathrm{s}\right)\end{array}$ \\
\hline 10 & 1.02 & 1.37 & 1.92 & 3.04 & 0.79 & 1.66 \\
\hline 17.5 & 0.78 & 1.08 & 1.32 & 2.21 & & \\
\hline 25 & 0.65 & 0.87 & 1.10 & 1.83 & 0.55 & 1.16 \\
\hline 37.5 & 0.53 & 0.69 & 0.90 & 1.55 & & \\
\hline 50 & 0.41 & 0.58 & 0.82 & 1.27 & 0.32 & 0.67 \\
\hline 62.5 & 0.35 & 0.48 & 0.59 & 1.00 & 0.25 & 0.53 \\
\hline 75 & 0.30 & 0.40 & 0.57 & 0.89 & 0.22 & 0.45 \\
\hline 87.5 & 0.25 & 0.33 & 0.48 & 0.73 & 0.18 & 0.38 \\
\hline 100 & 0.20 & 0.27 & 0.40 & 0.60 & $0.13^{\mathrm{a}}$ & $0.27^{\mathrm{a}}$ \\
\hline
\end{tabular}

${ }^{\text {a}}$ Value extrapolated to $\Phi_{t}=100 \%$.

using an ansatz for the entanglement segment number $N_{e}$ within the binary contact model, ${ }^{38}$

$$
N_{e} \propto \Phi^{\alpha} \text { with } \alpha=1 \text {. }
$$

Such a relation is suggested by previous measurements ${ }^{21}$ on high molecular weight $\mathrm{PE}$ in a oligomer matrix $\left(\mathrm{C}_{19} \mathrm{D}_{40}\right)$ where $\alpha=1.1$ was found. Despite the low molecular weights of the tracers, the description of the data at high tracer concentration is excellent. To achieve agreement with the absolute values of the PFG-NMR results $D\left(\Phi_{t}=100 \%\right)$ was taken as a fit parameter. The fitted value for $D(100 \%)$ deviates from the measured PFG-NMR result by $10 \%$, clearly within the experimental error of the latter. At low tracer fraction, however, Eq. (27) together with Eq. (28) overestimates the concentration dependence of the diffusion coefficients. We must bear in mind that Eq. (28) is based on a scenario in which the embedding matrix does not give any contribution to the entanglement formation. This is not the case for a matrix with a molecular weight at the entanglement threshold as investigated here. Richter et al. show ${ }^{15}$ that the relaxation rates $W_{p}$ for a monomodal polyethylene melt equivalent to our matrix are found, as expected from the Rouse model, to be independent of the mode number $p$ but the center-of-mass diffusion coefficient is already reduced by a factor 2 in comparison with the Rouse value. Also we must

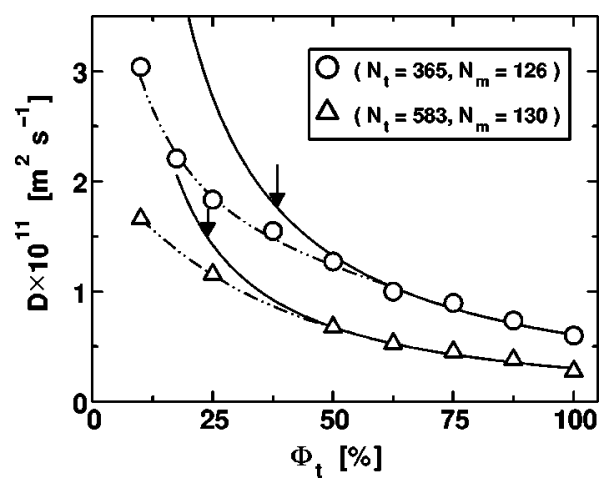

FIG. 3. PFG-NMR results for center-of-mass diffusion coefficient for both investigated systems. The solid lines show the results obtained from Eq. (27) with $N_{e} \propto \Phi_{t}^{-1}$ for the reptation diffusion coefficient. The condition $N_{e}\left(\Phi_{t}\right) / N_{t}=1$ is marked by arrows. The dashed-dotted lines are just a guide to the eye.

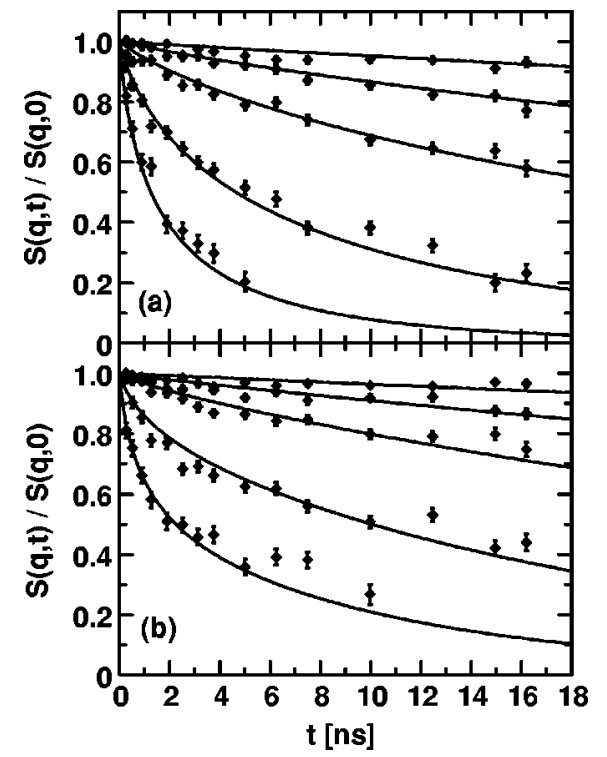

FIG. 4. NSE spectra for the system $\left(N_{t}=583, N_{m}=130\right)$ at a tracer concentration (a) $10 \%$ and (b) $87.5 \%$. The solid lines display the results of a fit within the mode analysis. The momentum transfers from top to bottom are $q=(0.037,0.055,0.077,0.115$, and 0.155$) \AA^{-1}$.

take into account that the ansatz discussed here is meaningless if the $\Phi_{t}$-dependent entanglement molecular weight becomes larger than the molecular weight of the tracers. From Eq. (28) it follows that

$$
\frac{N_{e}\left(\Phi_{t}^{\star}\right)}{N_{t}}=1 \Leftrightarrow \Phi_{t}^{\star}=\frac{N_{e}\left(\Phi_{t}=100 \%\right)}{N_{t}} .
$$

This condition is marked in Fig. 3 by arrows.

These first qualitative results indicate that the distance between entanglements increases if the tracer concentration is decreased. The concentration dependence is not as strong as predicted by the binary contact model for entanglement formation (see Ref. 21, and references therein). We will discuss our results for $N_{e}\left(\Phi_{t}\right)$ within the framework of a slightly modified model after Kavassalis and Noolandi, ${ }^{18,19}$ a model for entanglement formation that takes chain-end effects into account.

\section{B. NSE results: Mode analysis}

In Figs. 4 and 5 the NSE spectra for both investigated systems for the lowest $\left(\Phi_{t}=10 \%\right)$ and highest $\left(\Phi_{t}\right.$ $=87.5 \%$ ) tracer concentrations are presented. The solid lines give the results of a fit within the mode analysis. A comparison of the spectra shows that the relaxation at high tracer fractions is strongly slowed down relative to lower tracer concentrations. For example, in the case of the system $\left(N_{t}\right.$ $\left.=365, N_{m}=126\right)$ the structure factor for $q=0.077 \AA^{-1} \mathrm{de}$ cays at the low tracer concentration to a value of $\approx 0.55$ in the experimental time range, whereas at high tracer fraction the decay only takes place to a value of $\approx 0.7$.

Here we analyze the data in terms of the mode analysis introduced in Sec. II A which provides separate access to the normal modes of the internal chain dynamics each describing an independent motion on a different length scale. In Figs. 4 

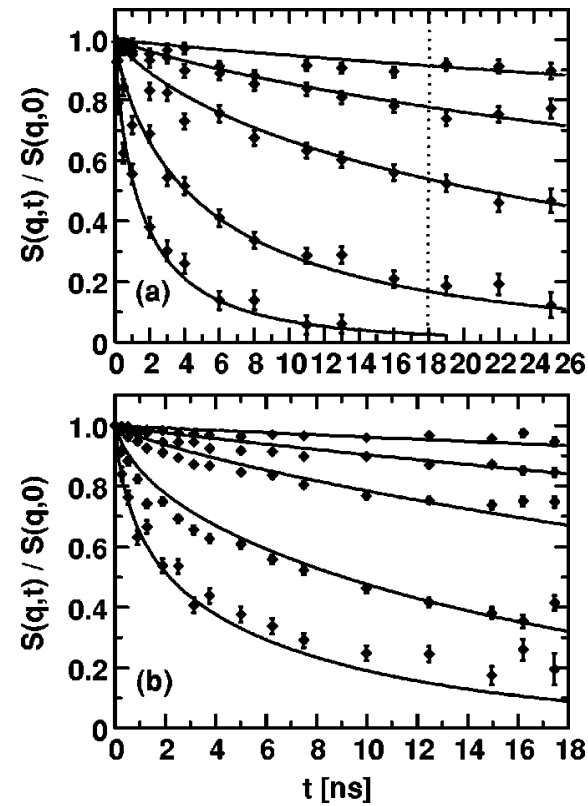

FIG. 5. NSE spectra for the system $\left(N_{t}=365, N_{m}=126\right)$ at a tracer concentration (a) $10 \%$ and (b) $87.5 \%$. The solid lines show the results of a fit within the mode analysis. Notice: the smaller time window of the Saclay experiment is marked by a dotted line in (a) (Jülich). The momentum transfers from top to bottom are $q=(0.037,0.055,0.077,0.115$, and 0.155$) \AA^{-1}$.

and 5 the results of the mode analysis fits are compared with the measured spectra. We fitted for each sample all experimental $q$-values simultaneously.
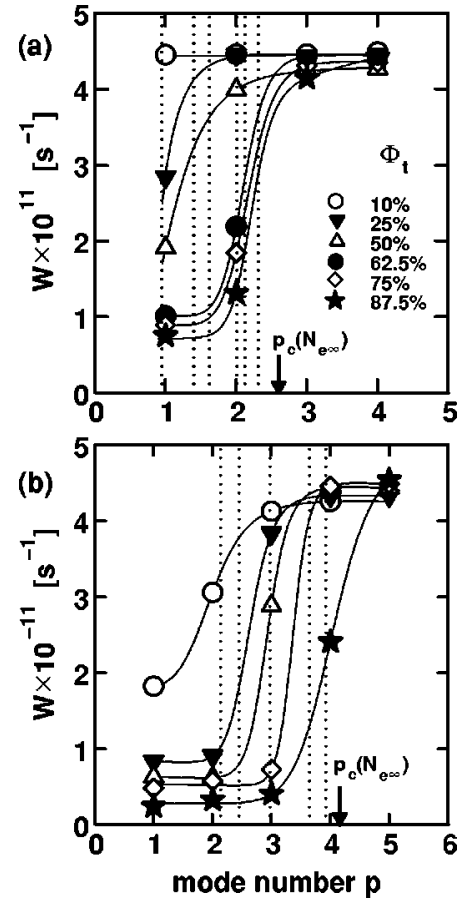

FIG. 6. Relaxation rates $W(p)$ obtained from a fit of the NSE spectra within the mode analysis versus mode number $p$ for the system (a) $\left(N_{t}=365, N_{m}\right.$ =126) and (b) $\left(N_{t}=583, N_{m}=130\right)$. The arrows mark the condition $p_{c}$ $=N_{t} / N_{e \infty}$. The dotted lines show the limiting mode numbers $p_{c}$ $=N_{t} / N_{c}\left(\Phi_{t}\right)$ from left to right with increasing tracer concentration. The $N_{c}\left(\Phi_{t}\right)$ are calculated from PFG-NMR diffusion coefficients via the Hess theory [Eq. (21)].
TABLE IV. Mode-dependent relaxation rates as obtained from a fit of the spectra within the mode analysis for the system $\left(N_{t}=365, N_{m}=126\right)$ and $\left(N_{t}=583, N_{m}=130\right)$

\begin{tabular}{|c|c|c|c|c|c|c|c|c|c|}
\hline \multirow{3}{*}{$\begin{array}{l}\text { System: } \\
\text { Mode } p: \\
\Phi_{t}(\%)\end{array}$} & \multicolumn{4}{|c|}{$\left(N_{t}=365, N_{m}=126\right)$} & \multicolumn{5}{|c|}{$\left(N_{t}=583, N_{m}=130\right)$} \\
\hline & 1 & 2 & 3 & 4 & 1 & 2 & 3 & 4 & 5 \\
\hline & \multicolumn{4}{|c|}{$W\left(10^{-11} \times 1 / \mathrm{s}\right)$} & \multicolumn{5}{|c|}{$W\left(10^{-11} \times 1 / \mathrm{s}\right)$} \\
\hline 10 & 4.5 & 4.5 & 4.5 & 4.5 & 1.8 & 3.1 & 4.1 & 4.3 & 4.3 \\
\hline 25 & 2.8 & 4.5 & 4.4 & 4.5 & 0.8 & 0.9 & 3.8 & 4.3 & 4.3 \\
\hline 50 & 1.9 & 4.0 & 4.3 & 4.3 & 0.6 & 0.6 & 2.9 & 4.4 & 4.4 \\
\hline 62.5 & 1.0 & 2.2 & 4.4 & 4.4 & 0.5 & 0.6 & 0.8 & 4.5 & 4.5 \\
\hline 75 & 0.9 & 1.8 & 4.3 & 4.4 & 0.5 & 0.6 & 0.7 & 4.5 & 4.5 \\
\hline 87.5 & 0.7 & 1.3 & 4.1 & 4.2 & 0.2 & 0.3 & 0.4 & 2.4 & 4.5 \\
\hline
\end{tabular}

In Fig. 6 the fit results for the relaxation rates $W_{p}$ are plotted as a function of the mode number $p$ and Table IV summarizes the results obtained. Obviously with increasing tracer concentration a successive slowing down of modes with low mode numbers $p$ (large length scales) is observed whereas the relaxation rates of the high numbered modes (small length scales) are unaffected. For the system with the lower tracer molecular weight $\left(N_{t}=365\right)$ at the lowest tracer concentration $\Phi_{t}=10 \%$, mode-independent relaxation rates are obtained. Here the Rouse model is valid. In contrast, for the tracer dynamics in the system $\left(N_{t}=583, N_{m}=126\right)$ even at $\Phi_{t}=10 \%$ severe deviations from the Rouse behavior are found. Apparently the relaxation of the first two modes is already hindered by topological constraints. Measurements on monomodal melts with molecular weights in the same range have shown that the only modes affected are those describing chain motions on length scales exceeding the entanglement distance determined for long chains. Having in mind that a mode $p$ describes a motion involving $N / p$ bonds we define a limiting mode number $p_{c}$ for which the spatial extent equals the length of a single entanglement strand,

$$
p_{c}=\frac{N_{t}}{N_{e}} \text {. }
$$

The limiting mode number $p_{c \infty}$ calculated from the longchain value of the entanglement segment number $N_{e \infty}=140$ is marked in Fig. 6 by arrows. In agreement with the results obtained for monomodal melts, modes are only slowed down if their mode numbers are smaller than $p_{c \infty}$ or in other words, if their spatial extents are larger than the entanglement distance in the long-chain limit. But now we find a concentration-dependent boundary between those modes affected and those unaffected by topological constraints. Consequently we have to introduce a $\Phi_{t}$-dependent limiting mode number

$$
p_{c}\left(\Phi_{t}\right)=\frac{N_{t}}{N_{e}\left(\Phi_{t}\right)},
$$

implying that the entanglement segment number must be a function of the tracer fraction. The strength of the topological constraints decreases with decreasing tracer concentration.

Besides the mode-dependent relaxation rates, a fit of the NSE spectra within the mode analysis includes the center-of- 


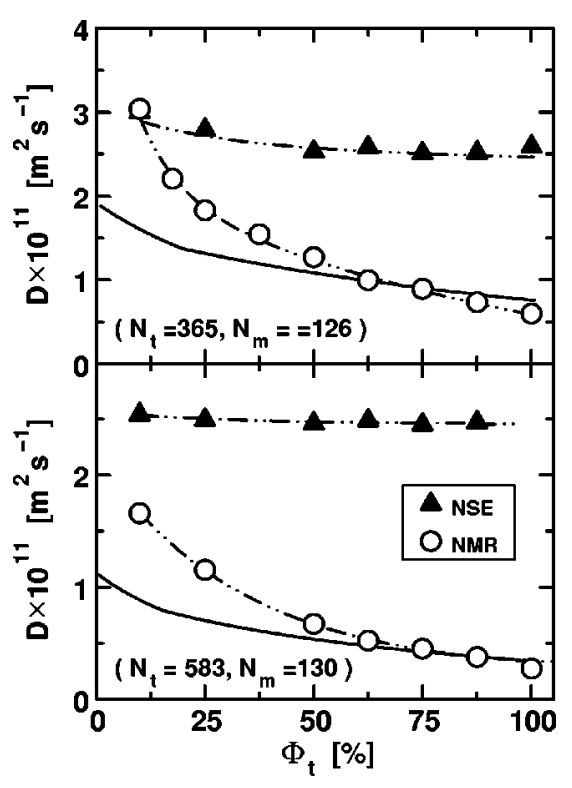

FIG. 7. Comparison between the measured PFG-NMR diffusion coefficients and the values obtained from a fit of the NSE spectra within the mode analysis for both investigated systems. The solid lines show the longtime diffusion coefficient calculated from the Hess theory with $N_{c}=150=$ constant [Eq. (21)].

mass diffusion coefficients of the tracers. In Fig. 7 the fit results $D_{\mathrm{NSE}}$ are compared with the $D_{\mathrm{NMR}}$ values measured by PFG-NMR. In contrast to the measured long-time diffusion coefficients the short-time diffusion coefficients $D_{\mathrm{NSE}}$ display only a weak dependence on the tracer concentration. In comparison to the NSE values the PFG-NMR results are systematically shifted to smaller values and the difference increases with increasing fraction of long tracer chains. Only in the case of the system with the lower tracer molecular weight $\left(N_{t}=365\right)$ at the lowest investigated tracer concentration $\Phi_{t}=10 \% D_{\mathrm{NSE}}$ equals $D_{\mathrm{NMR}}$. Considering that NSE measures the short-time behavior (ns) whereas the PFGNMR measures the value in the long-time limit (ms), these results suggest an effective time-dependent diffusion coefficient as obtained in the reptation model, as well as in the Hess model. Considering the relaxation time $\tau(p)$ of a mode $p$

$$
\tau(p)=\frac{N_{t}^{2}}{\pi^{2} p^{2} W(p)}
$$

we find further that the slowest relaxation time $\tau(1) \approx 30 \mathrm{~ns}$ for the system $\left(N_{t}=365, N_{m}=126\right)$ at $\Phi_{t}=10 \%$ is in the same range as the time window of the NSE experiment, while for all other concentrations, $\tau(1)$ is always much longer. For example, $\tau(1)$ is $193 \mathrm{~ns}$ for a tracer concentration of $\Phi_{t}=87.5 \%$.

These observations indicate that deviations between $D_{\mathrm{NSE}}$ and $D_{\mathrm{NMR}}$ do occur if the observation time in the NSE experiment is shorter than the relaxation time $\tau(p=1)$ of the slowest mode. At times $t<\tau(1)$ the center-of-mass diffusion is enhanced compared with the long-time value.

\section{Interpretation within the Hess model}

While the mode analysis assumes mode-dependent friction coefficients which themselves are constant with time, the essence of the Hess model is a length-scale independent but time-dependent monomeric friction. In the following we describe the NSE data in terms of this approach applying the generalization introduced in Sec. II B.

The parameters of the Hess theory are the Rouse rate $W_{R}$, the range of the effective excluded-volume interaction $q_{c}^{-1}$ and the critical number of segments $N_{c}$. We calculated the Rouse rate $W_{R}$ for each tracer concentration as described in the Appendix and kept its value fixed in the fit. The range of the excluded-volume interaction should be a material constant that depends only on the average distance $s$ between two chains $-s$ is given by the density of the polymer. Measurements on polyethylene melts gave a value of $q_{c}^{-1}$ $\approx 8 \AA$ which is of the order of the average distance $s$ $=m_{0}\left(l_{0} \varrho N_{A}\right)^{-1} \approx 5 \AA$ between PE chains at $T=509 \mathrm{~K}$. Here $N_{A}$ is the Avogadro number, $m_{0}$ the monomer weight, and $\varrho=0.733 \mathrm{~g} / \mathrm{cm}^{3}$ is the density of PE at $509 \mathrm{~K}$. The density of the sample does not change with molecular weight and therefore $q_{c}^{-1}$ should not vary with the tracer concentration.

The critical segment number was calculated by Hess in the limit of long chains neglecting chain-end effects. All segments are assumed to be statistically equivalent, in that inner as well as outer segments give the same contribution to the entanglement formation. For melts with high molecular weight the average interaction energy $\varphi$ between two segments and hence also $N_{c} \propto 1 / \varphi$ should depend only on thermodynamic parameters of the system but not on the molecular weight of the polymers. Therefore $N_{c}$ should be independent of $\Phi_{t}$. The result obtained from measurements on monomodal melts ${ }^{15}$ indeed gives $N_{c}=150=$ constant. Our finding of a concentration-dependent $N_{c}=N_{c}\left(\Phi_{t}\right)$ will be discussed in more detail in Sec. IV D.

If we follow the above considerations and assume that the parameters are independent of $\Phi_{t}$, no satisfactory description of the NSE spectra can be achieved over the full concentration range. However, if we restrict ourselves to concentrations above $\Phi_{t}=50 \%$, then the theory is able to fit the data with one common set of parameters. Thus, we determined for both systems the parameters $N_{c}$ and $q_{c}^{-1}$ fitting all spectra in this concentration range simultaneously. This yields for the system $\left(N_{t}=365, N_{m}=126\right): N_{c}=161$ and $q_{c}^{-1}=8 \AA$ and for the system $\left(N_{t}=583, N_{m}=130\right): N_{c}$ $=158$ and $q_{c}^{-1}=9 \AA$.

Figures 8 and 9 show for both systems the best and the worst agreement between the NSE spectra and the theoretical predictions, calculated with the above parameters. The quality of the agreement for tracer concentration above $\Phi_{t}$ $=50 \%$ is comparable with that obtained for $\Phi_{t}=87.5 \%$, but for low tracer concentrations the experimental data systematically show a much faster decay than the curves calculated from the Hess model with $N_{c}=$ constant. This signifies that at low tracer concentrations the actual hindrance of the chain motion by topological constraints is much weaker than as- 


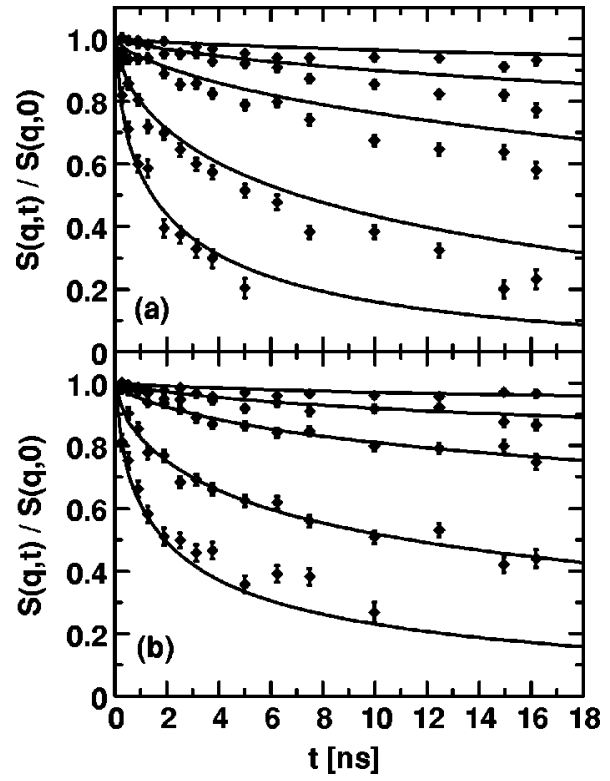

FIG. 8. $S(q, t)$ calculated within the Hess theory for the system $\left(N_{t}=583, N_{m}=130\right)$ at (a) $\Phi_{t}=10 \%$ (worst agreement) and (b) $\Phi_{t}$ $=87.5 \%$ (best agreement). The model parameters are $q_{c}^{-1}=9 \AA$ and $N_{c}$ $=158=$ constant. The momentum transfers are from top to bottom: $q$ $=(0.037,0.055,0.077,0.115$, and 0.155$) \AA^{-1}$.

sumed by the Hess model. The strength of the entanglement friction

$$
\frac{\Delta \zeta(t=0)}{\zeta} \propto \frac{1}{N_{c} q_{c}^{-1}}
$$
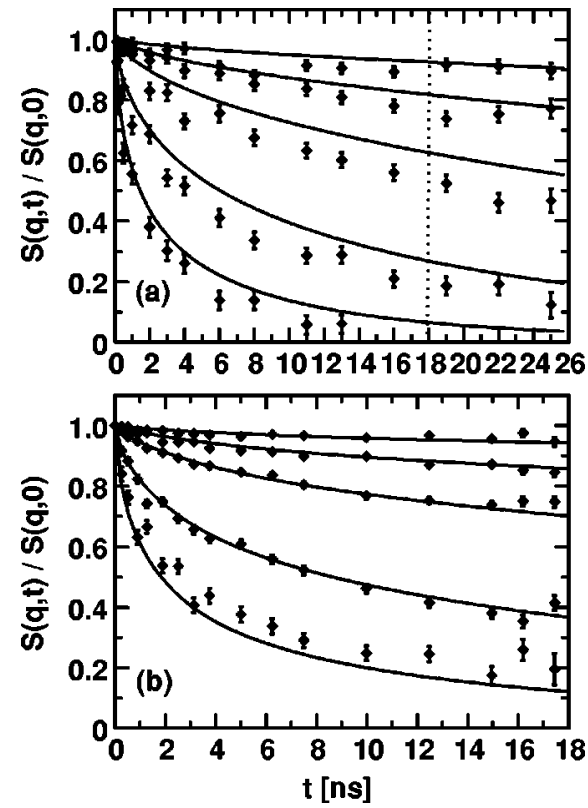

FIG. 9. $S(q, t)$ calculated within the Hess theory for the system $\left(N_{t}=365, N_{m}=126\right)$ at (a) $\Phi_{t}=10 \%$ (worst agreement) and (b) $\Phi_{t}$ $=87.5 \%$ (best agreement). The model parameter are $q_{c}^{-1}=8 \AA$ and $N_{c}$ $=161=$ constant. Notice: the smaller time window of the Saclay experiment (b) is marked by a dotted line in (a) (Jülich). The momentum transfers are from top to bottom: $q=(0.037,0.055,0.077,0.115$, and 0.155$) \AA^{-1}$.

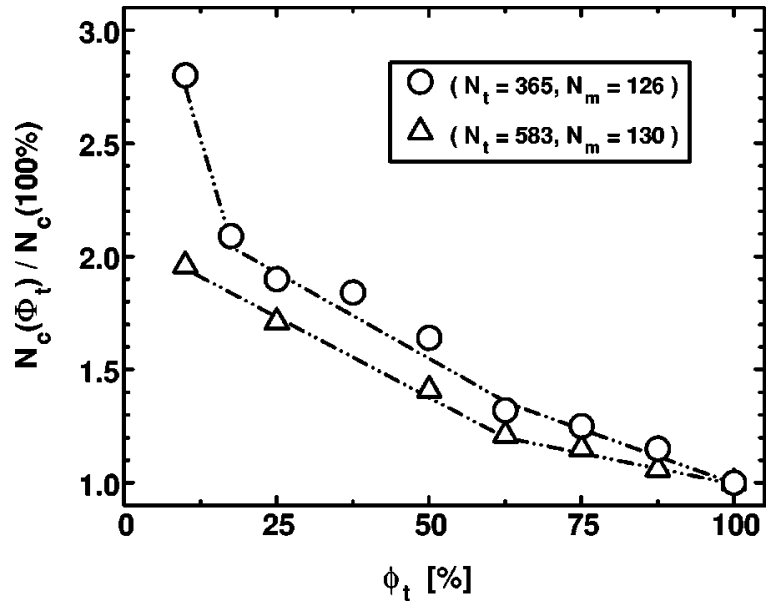

FIG. 10. Normalized critical segment number $N_{c}\left(\Phi_{t}\right) / N_{c}(100 \%)$ calculated within the Hess model [Eq. (21)] from the measured PFG-NMR diffusion coefficients for both investigated systems. The dashed-dotted lines are just a guide to the eye.

may be reduced by either increasing the range of the excluded-volume interaction or by an increase of the critical segment number with decreasing tracer fraction.

We now turn to the diffusion coefficients. The Hess theory predicts a time-dependent diffusion coefficient which in the short-time limit equals the Rouse value and therefore is expected, apart from free volume effects $D_{R} \propto 1 / \zeta$, to be independent of the tracer concentration. This is in agreement with the concentration-independent $D_{\mathrm{NSE}}$ as obtained from the mode analysis. Within the Hess model the long-time diffusion coefficient on the other hand is a function of the tracer concentration. This principal behavior is in qualitative agreement (see below) with the data measured with PFG-NMR. Figure 7 shows a comparison of the PFG-NMR results with the longtime diffusion coefficient calculated within the framework of the Hess theory with the parameters obtained from the fit described before. Obviously the concentration dependence of the calculated long-time diffusion coefficients is much too weak to describe the experimental data. In the Hess model following Eq. (21) the long-time diffusion coefficient is only a function of the critical segment number but does not depend on the range of the excluded-volume interaction.

We may now go backwards and calculate $N_{c}$ from the experimentally determined diffusion coefficients $D_{\mathrm{NMR}}$ using Eq. (21). In Fig. 10 the results for $N_{c}\left(\Phi_{t}\right)$, normalized to its value at $\Phi_{t}=100 \%$, are displayed as a function of the tracer concentration. In Table V the absolute values are summa-

TABLE V. Summary of the $N_{c}\left(\Phi_{t}\right)$ calculated within the Hess theory from the PFG-NMR data and the average range of the excluded-volume interaction $\left\langle q_{c}^{-1}\right\rangle$ obtained from a single fit of the NSE spectra of each system within the Hess model keeping $N_{c}\left(\Phi_{t}\right)$ fixed.

\begin{tabular}{cccccccc}
\hline \hline & \multicolumn{8}{c}{$\Phi_{t}(\%)$} & \\
\cline { 2 - 7 } System & 10 & 25 & 50 & 62.5 & 75 & 87.5 & $q_{c}^{-1}(\AA)$ \\
\hline$\left(N_{t}=365, N_{m}=126\right)$ & 384 & 261 & 225 & 181 & 172 & 158 & $7 \pm 9 \%$ \\
$\left(N_{t}=583, N_{m}=130\right)$ & 273 & 238 & 196 & 168 & 160 & 149 & $9 \pm 14 \%$ \\
\hline \hline
\end{tabular}




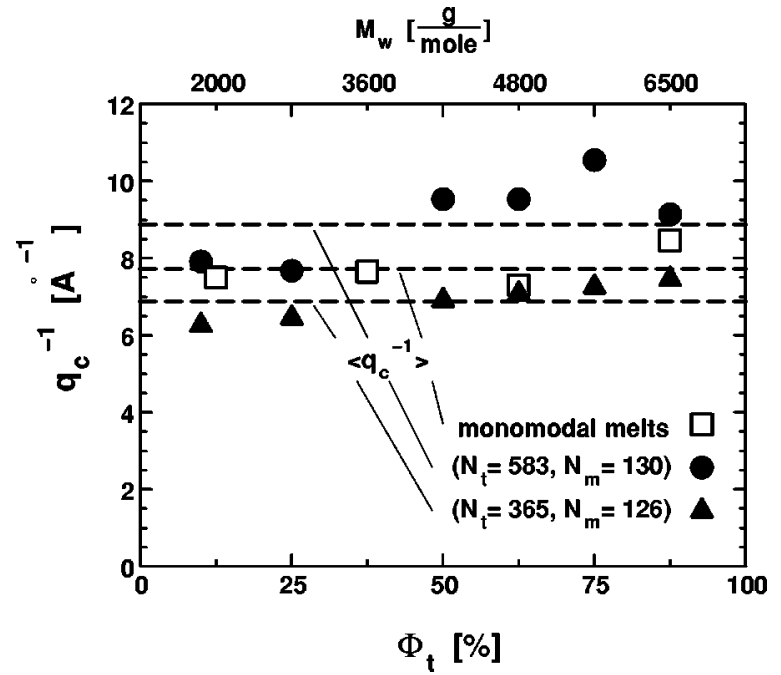

FIG. 11. Range of the excluded-volume interaction $q_{c}^{-1}\left(\Phi_{t}\right)$ as obtained from separate fits (a) for the bimodal melts keeping the $N_{c}\left(\Phi_{t}\right)$ determined from the PFG-NMR diffusion coefficients fixed (Table V) (b) for the monomodal melts investigated by Richter et al. (Ref. 15) with $N_{c}=150$. The average value $\left\langle q_{c}^{-1}\right\rangle$ for each system is marked by dashed lines.

rized. Down to a tracer concentration of $\Phi_{t}=25 \%$, the increase of $N_{c}\left(\Phi_{t}\right) / N_{c}(100 \%)$ is of the same order for both systems. The relative increase of $N_{c}$ for the system $\left(N_{t}\right.$ $=583, N_{m}=130$ ) amounts to a factor of 2 over the full concentration range. At the lowest investigated tracer concentration $\left(\Phi_{t}=10 \%\right)$ for the system with the shorter tracer chains a much larger value for $N_{c}\left(\Phi_{t}\right) \approx 390>N_{t}=365$ is obtained. Remembering the definition of the limiting mode number $p_{c}=N_{t} / N_{c}\left(\Phi_{t}\right)$ - here smaller than 1-the dynamics of the tracer chains should show Rouse behavior.

With these results we now may come back to the mode analysis results and calculate refined limiting mode numbers on the basis of $N_{c}\left(\Phi_{t}\right)$. These are marked in Fig. 6 by vertical dotted lines. The $p_{c}\left(\Phi_{t}\right)$ calculated via a theoretical approach that is completely independent of the mode analysis and obtained from a completely different set of (macroscopic) data provides an excellent description of the boundary between unaffected and hindered modes.

Last but not least, the NSE spectra for each concentration were fitted for both systems with $q_{c}^{-1}$ as the only parameter. The $N_{c}\left(\Phi_{t}\right)$ (see Table $\mathrm{V}$ ) were fixed to the values calculated from the PFG-NMR diffusion coefficients. In Fig. 11 the resulting $q_{c}^{-1}\left(\Phi_{q}\right)$ are plotted vs the tracer concentration $\Phi_{t}$ for each system. The results for the monomodal melts investigated by Richter $e t$ al. ${ }^{15}$ are shown as a function of the molecular weight of the polymers. The maximal deviation of $q_{c}^{-1}\left(\Phi_{t}\right)$ determined in separate fits from the average value $\left\langle q_{c}^{-1}\right\rangle$ (dashed lines) are $9 \%$ for the monomodal melts as well as for the system $\left(N_{t}=365, N_{m}=126\right)$ and $14 \%$ for the system $\left(N_{t}=583, N_{m}=130\right)$, respectively. The differences from the value obtained for the monomodal melts $q_{c}^{-1}=8 \AA$ are within the experimental error.

Figures 12 and 13 show the excellent agreement between the NSE spectra and the prediction of the Hess theory calculated with the parameters summarized in Table V; $N_{c}\left(\Phi_{t}\right)$

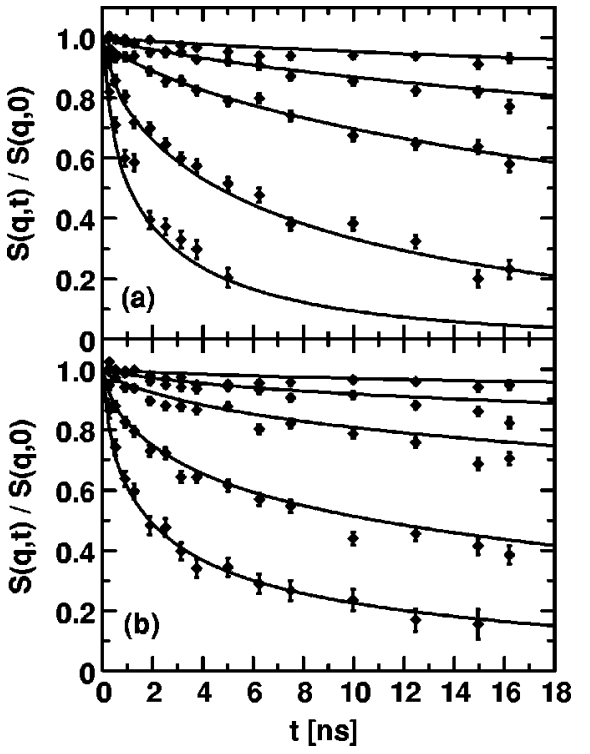

FIG. 12. $S(q, t)$ calculated within the Hess theory for the system $\left(N_{t}\right.$ $=583, N_{m}=130$ ) at (a) $\Phi_{t}=10 \%$ (best agreement) and (b) $\Phi_{t}=87.5 \%$ (worst agreement). The model parameter are taken from Table V. The momentum transfers are from top to bottom: $q=(0.037,0.055,0.077,0.115$, and 0.155$) \AA^{-1}$.

and the average range of excluded-volume interaction $\left\langle q_{c}^{-1}\right\rangle$.

In summary, with the critical segment numbers $N_{c}\left(\Phi_{t}\right)$ calculated from the PFG-NMR diffusion coefficients, the Hess model describes the NSE spectra of both systems very well, thus combining results from a macroscopic and a microscopic experiment in a consistent way.
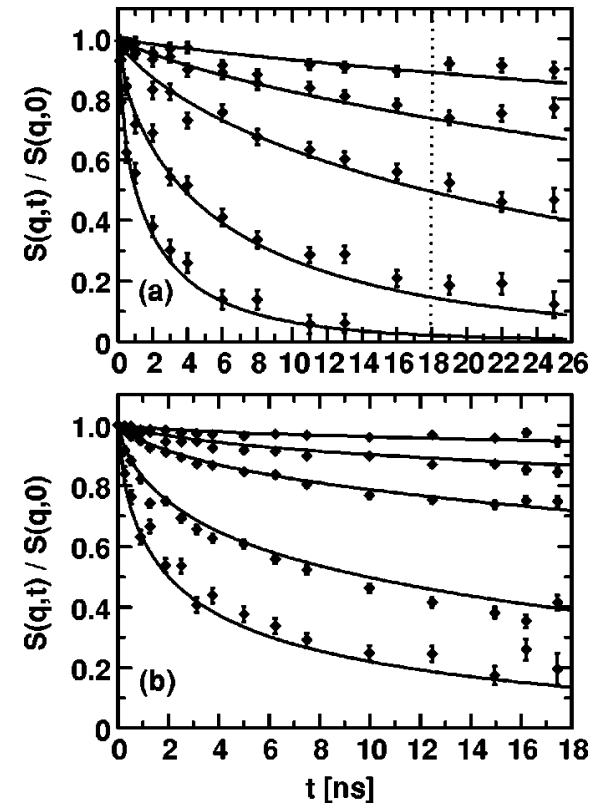

FIG. 13. $S(q, t)$ calculated within the Hess theory for the system $\left(N_{t}\right.$ $=365, N_{m}=126$ ) at (a) $\Phi_{t}=10 \%$ (best agreement) and (b) $\Phi_{t}=87.5 \%$ (worst agreement). The model parameter are taken from Table V. Notice: the smaller time window of the Saclay experiment is marked by a dotted line in (a) (Jülich). The momentum transfers are from top to bottom: $q$ $=(0.037,0.055,0.077,0.115$, and 0.155$) \AA^{-1}$. 


\section{Chain-end effects}

The observed increase of the entanglement segment number with decreasing tracer concentration cannot be explained in terms of conventional models for the entanglement formation. The actual $\Phi_{t}$ dependence is much weaker than theoretically predicted. The reason for this shortcoming lies in the fact that in these models (1) the matrix is assumed not to contribute to the entanglement formation and (2) long chains are only considered. In these models the probability for the formation of an entanglement is constant as long as the segment density does not change and hence $N_{e}$ should not depend on the composition in a bimodal melt. In our case neither condition (1) nor (2) is fulfilled. The matrix contributes to the entanglement formation and the molecular weight of the tracer is not in the long-chain limit.

The packing model by Kavassalis and Noolandi ${ }^{18,19}$ is an attempt to take the chain-end effects into account. Because of their higher degree of freedom, the chain ends give a weaker or no contribution to the entanglement formation than do inner segments. The number of end segments increases relative to the number of inner segments with decreasing molecular weight of the polymer. Therefore the probability for entanglement formation will be a function of the degree of polymerization, i.e., $N_{e}$ increases with decreasing $M_{w}$, and consequently $N_{e}$ will also depend on the composition of a bimodal melt.

Starting from the assumption that for the formation of a single entanglement strand of length $d_{e}$ a certain number of chains have to go through a test volume $V_{e} \propto d_{e}^{3}$, this volume can be expressed in terms of the average number $N(m)$ $=N_{\text {int }}(m)+N_{\text {end }}(m)$ of chains with exactly $m$ segments in $V_{e}$,

$$
V_{e}=l^{3}\left[N_{e}+\sum_{m=1}^{N} N_{\text {end }}(m) m+\sum_{m=1}^{N} N_{\text {int }}(m) m\right] .
$$

Here $l^{3}$ is the volume of a single segment and the subscripts (int) and (end) distinguish the contributions of internal segments and chain ends to $N(m)$. As a model parameter the so-called coordination number,

$$
\widetilde{N}=N_{e}^{-1} \sum_{m=1}^{N} N_{\text {int }}(m) m
$$

is introduced summarizing the contribution of the internal segments. For a homogeneous distribution of segments, $N_{\text {int }}$ is replaced by a product of the average number of chain ends $\left\langle N_{\text {end }}\right\rangle=2 \varrho V_{e} / N$ and the average number of segments $\left\langle m_{\mathrm{end}}\right\rangle$ in $V_{e}$. Assuming a Gaussian correlation between the ends of a single chain the authors give as an estimate for the length of a chain end $\left\langle m_{\text {end }}\right\rangle \approx N_{e} / 2$, leading to the final result for $N_{e}$,

$$
\widetilde{N}+1=\frac{\pi}{6} \sqrt{N_{e}}\left(1-N_{e} / N\right)
$$

The coordination number introduced above is well defined by the long-chain limit of $N_{e \infty}=N_{e}(N \rightarrow \infty) \approx 140$ for PE at $T=509 \mathrm{~K}$, giving a value of

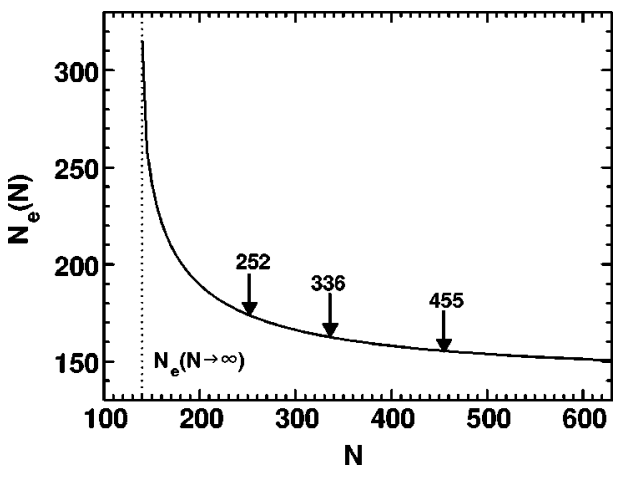

FIG. 14. $N_{e}(N)$ for monomodal melts as a function of the polymer segment number $N$ calculated from Eq. (37). The dotted line show the value for $N_{e}$ $\approx 140$ in the long-chain limit at $T=509 \mathrm{~K}$ (Refs. 21 and 24). The arrows mark the segment numbers of the samples investigated by Richter et al. (Ref. 15).

$$
\widetilde{N}=\frac{\pi}{6} \sqrt{N_{e^{\infty}}}-1 \approx 5 .
$$

Below a certain degree of polymerization, $N_{k}$ $=\left(243 / \pi^{2}\right)(\tilde{N}+1)^{2}$, solutions for $N_{e}(N)$ are no longer obtained (see also Fig. 14). This has been interpreted by the authors as a transition between an entangled and an unentangled state of the melt. Following Eq. (37) this transition takes place at a degree of polymerization 6 times larger than $N_{e \infty}$ showing that this theory obviously highly overestimates the effective length of the chain ends.

Assuming now that the proportionality of $\left\langle m_{\text {end }}\right\rangle$ to $N_{e}$ is still valid but the effective length of the chain ends is different from $N_{e} / 2$, we can determine with the condition $N_{k}$ $\approx N_{e \infty}$ the factor

$$
\epsilon=\frac{\pi^{2}}{243} \frac{N_{e \infty}}{(\widetilde{N}+1)^{2}} \approx 0.15,
$$

that leads to a reduction of the effective length of an entanglement. Note that $\epsilon$ is well defined by measurable quantities. This modification yields the final result

$$
\widetilde{N}+1=\frac{\pi}{6} \sqrt{N_{e}}\left(1-\epsilon N_{e} / N\right)
$$

or generalized to a bimodal melt,

$$
\widetilde{N}+1=\frac{\pi}{6} \sqrt{N_{e}}\left(1-\epsilon N_{e}\left[\Phi_{t} / N_{t}+\left(1-\Phi_{t}\right) / N_{m}\right]\right) .
$$

In Fig. $15 N_{e}\left(\Phi_{t}\right)$ is displayed as a function of the tracer concentration. The predicted values of $N_{e}\left(\Phi_{t}\right)$ calculated from Eq. (40) are extremely sensitive to the actual value of the matrix molecular weight relative to the exact value for $N_{e^{\infty}}$. As a consequence of the uncertainties in the PFGNMR values for the diffusion coefficients, in the determination of the molecular weights of the polymers and in the assignment of $N_{k}$ to $N_{e \infty}$, we do not expect quantitative agreement with the $N_{c}\left(\Phi_{t}\right)$ determined from the PFG-NMR data via the Hess theory. For this reason in Fig. 15 the results for two comparable matrix segment numbers of $N_{m} \pm 5$ are also shown. 


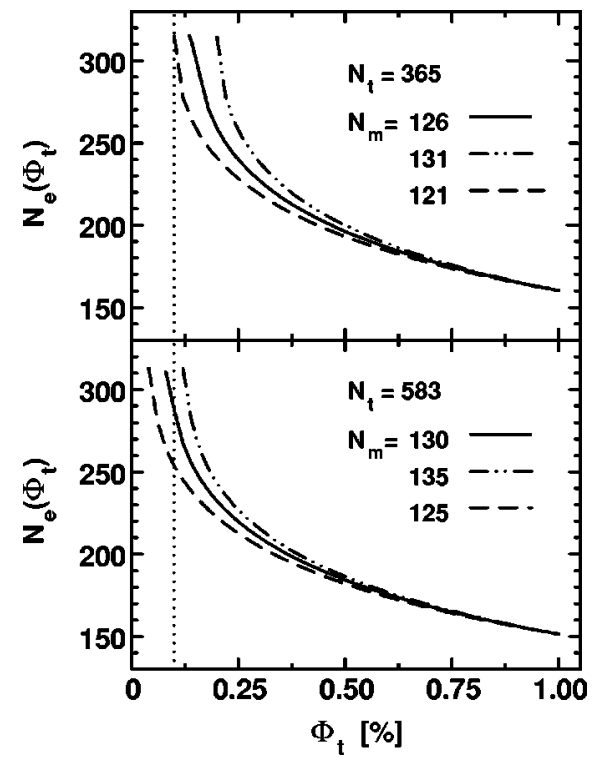

FIG. 15. $N_{e}\left(\Phi_{t}\right)$ as a function of the tracer concentration calculated from Eq. (40) for both investigated systems and two comparable matrix molecular weights. The dotted line marks the lowest investigated tracer concentration $\Phi_{t}=10 \%$.

Despite these restrictions, nearly quantitative agreement is achieved. Down to a tracer concentration of $\Phi_{t}=25 \% N_{e}$ increases in both systems about a factor $\approx 1.5$ in comparison to its value at $\Phi_{t}=100 \%$. This increase is just about $17 \%$ smaller than the increase obtained for the $N_{c}\left(\Phi_{t}\right)$. For the smallest investigated tracer concentration the system with the smaller tracer and matrix molecular weights is already in the unentangled state. This result is in agreement with the results from the Hess theory as well as with those obtained within the mode analysis; $p_{c}<1$ and hence the tracer should show Rouse behavior. The system $\left(N_{t}=583, N_{m}=130\right)$ at $\Phi_{t}$ $=10 \%$ appears to be at the boundary between the entangled and the unentangled state. In comparison to the system with the shorter tracer chains the transition is shifted to lower tracer concentration and with $N_{e}(10 \%) \approx 290$ we obtain a limiting mode number of $p_{c} \approx 2$. In agreement with the results of the mode analysis the tracers no longer show Rouse dynamics. Even at this low tracer concentration the internal dynamics are already hindered, resulting in a slowing down of the first two modes.

Thus the weakening of the topological constraints with decreasing tracer fraction can be well described by taking the influence of the chain ends on the entanglement formation into account. From Eq. (39) we can see that the entanglement segment number should also be a function of the molecular weight of the polymers. This contradicts the result $N_{e}=$ constant obtained from previous measurements on monomodal melts. Applying the refined Eq. (39) in Fig. 14, the entanglement segment number $N_{e}(N)$ is plotted vs the segment number $N$ and the chain lengths investigated in these earlier experiments are marked by arrows. $N_{e}$ should be nearly constant with a value of $160 \pm 10$ for the three highest measured molecular weights. The slightly increased average value for $N_{e}$ can be easily compensated by a slightly shorter range of the excluded-volume interaction. A measurable ef-

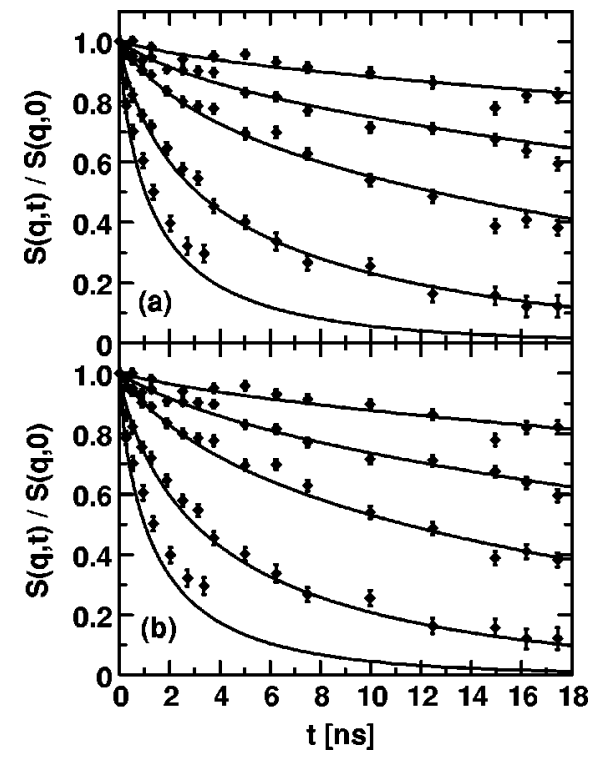

FIG. 16. $S(q, t)$ calculated within the Hess theory for the monomodal melt (a) $N=140$ with the parameters $\left(q_{c}^{-1}=8 \AA, N_{c}=150\right)$ and (b) $N=154$ with the parameters $\left(q_{c}^{-1}=8 \AA, N_{c}=230\right)$ in comparison with the experimental spectra. The momentum transfers are from top to bottom: $q$ $=(0.037,0.055,0.077,0.115$, and 0.155$) \AA^{-1}$.

fect should therefore only be seen for the sample with the smallest segment number $N \approx N_{e \infty} \approx 140$. As mentioned before the actual value for $N_{e}$ for polymers with $N \approx N_{e \infty}$ is extremely sensitive to the actual molecular weight of the sample and the correct value for $N_{k} \approx N_{e \infty}$. Realistic uncertainties of order $10 \%$ can affect the results strongly. For example, in Ref. 15 the molecular weights of the precursor polymers are not corrected for saturation. Figure 16 shows that under the assumption of a slightly increased molecular weight $N=140+10 \%$ and $N_{c}=230$ [obtained from Eq. (37)] a similar excellent description of the NSE spectra is achieved as with $N=140$ and $N_{c}=150$. Finally, we conclude that in our experiment on bimodal melts we are able to measure the influence of chain ends on $N_{e}$ because we measure the relative changes of $N_{e}$ to the value at $\Phi_{t}=100 \%$ and because the matrix chains investigated here are shorter than the shortest chains investigated in the previous experiments on monomodal polyethylene melts.

\section{CONCLUSION}

We have used neutron spin-echo spectroscopy to investigate on a microscopic length scale the influence of topological constraints on the single-chain dynamics in the transition region from Rouse- to reptationlike behavior. The transition region was covered by changing the fraction of long tracer molecules in a short-chain matrix over the full concentration range. By means of PFG-NMR, the long-time dynamics on a macroscopic length scale were explored.

A mode analysis allowed us to extract from the NSE spectra the relaxation times for the different normal modes of the chain dynamics each describing a motion on a different length scale. Similar to results from monomodal melts we saw a strong slowing down of those modes related to large length scale motions (low mode number $p$ ) of the chain. 
Modes are only affected, if their spatial extension exceeds the concentration-dependent length of a single entanglement strand $N_{e}$. The concentration dependence of the boundary between affected and unaffected modes as well as the strong variation of the measured diffusion coefficients suggest that the strength of the topological constraints decreases with decreasing tracer fraction, $N_{e}=N_{e}\left(\Phi_{t}\right)$. The concentration dependence is much weaker than predicted by conventional models for entanglement formation. We introduce a selfconsistent modification of the model formulated by Kavassalis and Noolandi. Within this model, the concentration dependence can be explained by taking into account the enhanced mobility of the chain ends in comparison to the inner segments. The number of end segments relative to the number of inner segments increases with decreasing tracer fraction, resulting in a weakening of the topological constraints.

The "short-time" center-of-mass diffusion coefficient obtained from the NSE data via the mode analysis is constant and systematically faster than the strong concentrationdependent long-time values measured with PFG-NMR. This indicates an effective time-dependent diffusion coefficient.

A microscopic model providing a time-dependent diffusion coefficient is the Hess model. We generalized the Hess model to polydisperse melts. Hess introduced the critical segment number $N_{c}$ as model parameter under the assumption that all segments are statistically equivalent; chain-end effects can be neglected. We therefore calculated the $N_{c}\left(\Phi_{t}\right)$ via the Hess theory from the PFG-NMR data and finally obtained an excellent description of our NSE spectra over the full concentration range. The $N_{c}\left(\Phi_{t}\right)$ and the $N_{e}\left(\Phi_{t}\right)$ calculated from the modified model after Kavassalis and Noolandi show excellent agreement. Last but not least, a comparison of the limiting mode numbers $p_{c}=N_{t} / N_{c}\left(\Phi_{t}\right)$ with the results from the mode analysis shows that the boundary between unhindered and already hindered modes can be described in the same framework.

We want to point out that we obtained a complete and consistent description for our macroscopic and microscopic, short- and long-time data. Both approaches - the Hess model as a microscopic ansatz and the mode analysis parameterizing only the entanglement effect—give compatible results.

\section{APPENDIX: CONCENTRATION DEPENDENCE OF $\zeta$}

Pearson et al. ${ }^{36,37}$ have measured the diffusion coefficients (PFG-NMR) and viscosities (capillar viscosemetry, rheology) of polyethylene melts over a wide range of molecular weights and temperatures. They found that the monomeric friction coefficient $\zeta$ follows a Vogel-FulcherThamann relationship,

$$
\zeta=\zeta_{\infty} \exp \left(\frac{B}{\Delta\left(T-T_{0}\right)}\right)
$$

with $B=0.6$ and the high temperature limit of $\zeta: \zeta_{\infty}=3.7$ $\cdot 10^{11} \mathrm{dyn} \mathrm{s} / \mathrm{cm}$. As a result of the increase of the free volume with decreasing molecular weight $\Delta \alpha$ and the critical temperature $T_{0}$ are functions of the molecular weight,

$$
\Delta \alpha=\alpha_{\infty}(1+\beta / M) \text { and } T_{0}=T_{0}^{\infty}(1+\gamma / M),
$$

with $\alpha_{\infty}=5.1 \cdot 10^{-4} 1 /{ }^{\circ} \mathrm{C}, \beta=160 \mathrm{~g} / \mathrm{mol}, T_{0}^{\infty}=160 \mathrm{~K}$, and $\gamma=80 \mathrm{~g} / \mathrm{mol}$.

With the high molecular weight limit of $W_{R}\left(M_{w}\right.$ $=70000 \mathrm{~g} / \mathrm{mol}$ ) at $T=509 \mathrm{~K}$ determined by Richter et al. ${ }^{21}$ to be $W_{R}=3.59 \cdot 10^{11} \mathrm{~s}^{-1}$ the Rouse rate $W_{R} \propto 1 / \zeta$ can be rescaled,

$$
W_{R}\left(T_{2}, M_{2}\right)=W_{R}\left(T_{1}, M_{1}\right) \frac{T_{2}}{T_{1}} \frac{\zeta\left(T_{1}, M_{1}\right)}{\zeta\left(T_{2}, M_{2}\right)} .
$$

For PE melts the monomeric friction coefficient varies about $30 \%$ in a molecular weight range of $M_{w}=(2000-70000)$ $\mathrm{g} / \mathrm{mol}$ resulting in a change of $W_{R}$ with the composition of the bimodal melt. The concentration dependence is calculated as follows:

$$
W_{R}^{\mathrm{bi}}=\Phi_{t} W_{R}^{t}+\Phi_{m} W_{R}^{m}
$$

Here the superscript bi stands for bimodal melt and the superscripts $m$ and $t$ mark the quantities belonging to the monomodal melts corresponding to the single components of the bimodal melt. The increase of the Rouse rate by lowering the tracer concentration from $100 \%$ to $10 \%$ is for the system $\left(N_{t}=583, N_{m}=130\right)$ and $\left(N_{t}=365, N_{m}=126\right)$ of about $20 \%$ and $17 \%$, respectively. The random phase approximation (RPA) theory ${ }^{39}$ would lead with

$$
\frac{1}{W_{R}^{\mathrm{bi}}}=\frac{\Phi_{t}}{W_{R}^{t}}+\frac{\Phi_{m}}{W_{R}^{m}}
$$

to slightly different values for $W_{R}^{\mathrm{bi}}$. In comparison to those obtained from Eq. (A4) the results change about $1.2 \%$ for the system $\left(N_{t}=583, N_{m}=130\right)$ and $0.8 \%$ for the system $\left(N_{t}\right.$ $=365, N_{m}=126$ ). These differences between both approaches can be neglected.

${ }^{1}$ P. G. de Gennes, Physica (Great Britain) 3, 37 (1967).

${ }^{2}$ P. G. de Gennes, J. Phys. (France) 42, 735 (1981).

${ }^{3}$ M. Doi and S. F. Edward, J. Chem. Soc., Faraday Trans. 1 74, 1789 (1978); 74, 1802 (1978); 74, 1818 (1978).

${ }^{4}$ G. Ronca, J. Chem. Phys. 79, 1031 (1983).

${ }^{5}$ J. de Cloizeaux, J. Phys. I 3, 1523 (1993).

${ }^{6}$ P. Schleger, B. Farago, C. Lartigue, A. Kollmer, and D. Richter, Phys. Rev. Lett. 81, 124 (1998).

${ }^{7}$ A. P. Chatterjee and R. F. Loring, J. Chem. Phys. 101, 1595 (1994).

${ }^{8}$ J. D. Wilson and R. F. Loring, J. Chem. Phys. 103, 1641 (1995).

${ }^{9}$ W. Hess, Macromolecules 19, 1395 (1986).

${ }^{10}$ W. Hess, Macromolecules 20, 2587 (1987).

${ }^{11}$ W. Hess, Macromolecules 21, 2620 (1988).

${ }^{12}$ K. S. Schweizer, J. Chem. Phys. 91, 5802 (1989).

${ }^{13}$ K. S. Schweizer, J. Chem. Phys. 91, 5822 (1989).

${ }^{14}$ K. S. Schweizer and G. Szamel, J. Chem. Phys. 103, 1934 (1995).

${ }^{15}$ D. Richter, L. Willner, A. Zirkel, B. Farago, L. J. Fetters, and J. S. Huang, Macromolecules 27, 7437 (1994)

${ }^{16}$ M. Fuchs and K. S. Schweizer, Macromolecules 30, 5133 (1997).

${ }^{17}$ M. Fuchs and K. S. Schweizer, Macromolecules 30, 5156 (1997).

${ }^{18}$ T. A. Kavassalis and J. Noolandi, Macromolecules 21, 2869 (1988).

${ }^{19}$ T. A. Kavassalis and J. Noolandi, Phys. Rev. Lett. 59, 2674 (1987).

${ }^{20} \mathrm{M}$. Doi and S.F. Edwards, The Theory of Polymer Dynamics (Clarendon Press, Oxford, 1986).

${ }^{21}$ D. Richter, B. Farago, B. Butera, L. J. Fetters, J. S. Huang, and B. Ewen, Macromolecules 26, 795 (1993).

${ }^{22}$ P. E. Rouse, J. Chem. Phys. 21, 1272 (1953).

${ }^{23}$ Recently published comparison between computer simulation results and NSE measurements question the strict validity of the Rouse model even in this limit (see Ref. 40).

${ }^{24}$ D. Richter, B. Butera, L. J. Fetters, J. S. Huang, B. Farago, and B. Ewen, Macromolecules 25, 6156 (1992). 
${ }^{25}$ A. R. Rennie, A. T. Boothroyd, and C. B. Boothroyd, Europhys. Lett. 15, 715 (1991).

${ }^{26}$ J. P. Hansen and I. R. McDonald, Theory of Simple Liquids, 2nd ed. (Academic, London, 1986), Chap. 9.

${ }^{27}$ J. des Cloizeaux, J. Europhys. Lett. 6, 437 (1988).

${ }^{28}$ J. des Cloizeaux, Macromolecules 23, 4678 (1990).

${ }^{29}$ J. des Cloizeaux, Macromolecules 25, 835 (1992).

${ }^{30}$ M. D. Gehlsen and F. S. Bates, Macromolecules 26, 4122 (1983).

${ }^{31}$ F. Mezei, The Principles of Neutron Scattering in Neutron Spin Echo, in Lecture Notes in Physics, edited by F. Mezei (Springer, Berlin, 1980).

${ }^{32} \mathrm{~B}$. Ewen and D. Richter, Neutron Spin Echo Investigations on the Segmental Dynamics of Polymers in Melts, Networks and Solutions, in Advances in Polymer Science (Springer, Berlin, 1997), Vol. 134.

${ }^{33}$ J. Kärger and G. Fleischer, TrAC, Trends Anal. Chem. 94, 145 (1994).
${ }^{34}$ P. T. Callaghan, Phys. Rev. Lett. 68, 3176 (1992).

${ }^{35}$ G. Fleischer and F. Fujara, NMR as a Generalized Incoherent Scattering Experiment, in NMR Basic Principles and Progress (Springer, Berlin, 1994).

${ }^{36}$ D. S. Pearson, L. J. Fetters, W. Graessley, G. Ver Strate, and E. von Meerwall, Macromolecules 27, 711 (1994).

${ }^{37}$ D. S. Pearson, G. Ver Strate, E. von Meerwall, and F. C. Schilling, Macromolecules 20, 1133 (1987).

${ }^{38}$ S. F. Edwards, Proc. Phys. Soc. London 92, 9 (1967).

${ }^{39}$ A. Z. Ackasu, Dynamic Scattering from Multicomponent Polymer Mixtures in Solution and Bulk, in Dynamic Light Scattering: The Method and Some Applications (Clarendon, Oxford, 1993).

${ }^{40}$ W. Paul, G. D. Smith, D. Y. Yoon, B. Farago, S. Rathgeber, A. Zirkel, L. Willner, and D. Richter, Phys. Rev. Lett. 80, 2346 (1998). 